\title{
BOUNDARY HARMONIC COORDINATES ON MANIFOLDS WITH BOUNDARY IN LOW REGULARITY
}

\author{
STEFAN CZIMEK
}

\begin{abstract}
In this paper, we prove the existence of $H^{2}$-regular coordinates on Riemannian 3-manifolds with boundary, assuming only $L^{2}$-bounds on the Ricci curvature, $L^{4}$-bounds on the second fundamental form of the boundary, and a positive lower bound on the volume radius.

The proof follows by extending the theory of Cheeger-Gromov convergence to include manifolds with boundary in the above low regularity setting. The main tools are boundary harmonic coordinates together with elliptic estimates and a geometric trace estimate, and a rigidity argument using manifold doubling. Assuming higher regularity of the Ricci curvature, we also prove corresponding higher regularity estimates for the coordinates.
\end{abstract}

\section{Contents}

1. Introduction 2

1.1. The Cheeger-Gromov theory of manifold convergence 3

1.2. Boundary harmonic coordinates 4

1.3. Overview of the paper 5

1.4. Acknowledgements 5

2. Notations, definitions and prerequisites 6

2.1. Basic notation 6

2.2. Function and tensor spaces $\quad 7$

2.2.1. Continuous and Hölder-continuous functions $\quad 7$

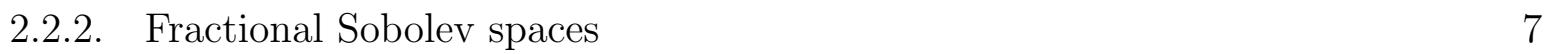

2.2.3. Tensor spaces 11

2.3. Riemannian geometry and boundary harmonic coordinates 11

2.4. The trace estimate for the Gauss curvature $K \quad 15$

3. The precise version of the main result $\quad 17$

4. Convergence of Riemannian manifolds with boundary 18

4.1. Convergence of functions, tensors and Riemannian manifolds 18

4.2. The $C^{m, \alpha}$-norm of a Riemannian manifold 19

4.3. The boundary harmonic $H^{2}$-norm of a Riemannian manifold 20

5. Existence of boundary harmonic coordinates 23

5.1. Existence of a converging subsequence in the pointed $C^{0, \alpha}$-topology for $\alpha \in(0,1 / 2) \quad 24$

5.2. Improvement to strong convergence in the pointed $H^{2}$-topology 26 
5.2.1. Strong convergence of the Gauss curvature 27

5.2.2. Strong convergence of $\not_{A B}$ and $g_{A B}$ components 27

5.2.3. Strong convergence of $g^{33}$ and $g^{3 A}$ components 28

5.3. Rigidity of the limit manifold $\quad 30$

5.3.1. Proof of smoothness of $g \quad 30$

5.3.2. The manifold double of $(M, g) \quad 30$

5.3.3. Conclusion of the rigidity of the limit manifold 32

5.4. The contradiction 33

6. Higher regularity estimates for boundary harmonic coordinates 33

Appendix A. Global elliptic estimates 38

A.1. Global estimates for Dirichlet data 38

A.2. Global estimates for Neumann data 41

Appendix B. Interior elliptic estimates $\quad 42$

References $\quad 43$

\section{INTRODUCTION}

This paper is concerned with the following question.

Consider a compact smooth Riemannian 3-manifold $(M, g)$ with boundary. Under which assumptions does there exist around each point in $M$ a local coordinate system $\left(x^{1}, x^{2}, x^{3}\right)$ of uniform size in which the metric components $g_{i j}$ are uniformly bounded in $\mathrm{H}^{2}$ ?

The following is a first version of our main theorem, answering the above question. The precise version is stated in Section 3.

Theorem 1.1 (Existence of regular coordinates, version 1). Let $(M, g)$ be a compact complete ${ }^{1}$ Riemannian 3-manifold with boundary such that

$$
\operatorname{Ric} \in L^{2}(M), \Theta \in L^{4}(\partial M), r_{v o l}(M, 1)>0,
$$

where $\Theta$ denotes the second fundamental form of $\partial M \subset M$ and $r_{\text {vol }}(M, 1)$ is the volume radius at scale 1 of $(M, g)^{2}$ Then the following holds.

(1) $L^{2}$-regularity. There is $\varepsilon_{0}>0$ such that for every $0<\varepsilon<\varepsilon_{0}$, there is a radius

$$
r=r\left(\|\operatorname{Ric}\|_{L^{2}(M)},\|\Theta\|_{L^{4}(\partial M)}, r_{v o l}(M, 1), \varepsilon\right)>0
$$

\footnotetext{
${ }^{1} \mathrm{~A}$ smooth Riemannian manifold with boundary is called complete if it is complete as a metric space.

${ }^{2}$ Let $(M, g)$ be a Riemannian 3-manifold. The volume radius at scale $r$ is defined as

$$
r_{v o l}(M, r):=\inf _{p \in M} \inf _{r^{\prime}<r} \frac{\operatorname{vol}_{g}\left(B_{g}\left(p, r^{\prime}\right)\right)}{\frac{4 \pi}{3}\left(r^{\prime}\right)^{3}},
$$

where $B_{g}\left(p, r^{\prime}\right)$ denotes the geodesic ball of radius $r^{\prime}$ centered at $p$.
} 
such that around every point $p \in M$ on a geodesic ball of radius $r$, there are coordinates $\left(x^{1}, x^{2}, x^{3}\right)$ in which

$$
(1-\varepsilon) e_{i j} \leq g_{i j} \leq(1+\varepsilon) e_{i j},
$$

where $e_{i j}$ denotes the Euclidean metric, and further

$$
\max _{i, j=1,2,3}\left(r^{-1 / 2}\left\|\partial g_{i j}\right\|_{L^{2}}+r^{1 / 2}\left\|\partial^{2} g_{i j}\right\|_{L^{2}}\right)<\varepsilon .
$$

(2) Higher regularity. Let $m \geq 1$ be an integer. Assuming higher regularity of Ric, we have further the higher regularity estimate

$$
\sum_{i, j=1,2,3}\left\|g_{i j}\right\|_{H^{m+2}} \leq C_{r} \sum_{i=0}^{m}\left\|\nabla^{(i)} \operatorname{Ric}\right\|_{L^{2}(M)}+C_{r, m} \varepsilon .
$$

Here, as in the rest of the paper, the notation is that a constant $C_{q_{1}, \ldots, q_{k}}>0$ depends on the quantities $q_{1}, \ldots, q_{k}$.

The proof of Theorem 1.1 is based on the so-called Cheeger-Gromov theory of manifold convergence. In the literature, the Cheeger-Gromov convergence theory and existence results for regular coordinates are readily available for

- manifolds without boundary in low regularity, see for example [20],

- manifolds with boundary in higher regularity, see for example [13], [4], [5] and [16]. These results assume pointwise bounds on Ric, while in Theorem 1.1 we only assume $L^{2}$-bounds on Ric.

Our main motivation to prove Theorem 1.1 comes from applications to the Cauchy problem of general relativity, see for example the localised bounded $L^{2}$-curvature theorem in [7].

The rest of this introduction is organised as follows. In Section 1.1, we introduce the Cheeger-Gromov theory of manifold convergence and give a short historic overview of its development. In Section 1.2, we discuss so-called boundary harmonic coordinates which are essential for our study of the Cheeger-Gromov theory for manifolds with boundary in low regularity.

1.1. The Cheeger-Gromov theory of manifold convergence. The Cheeger-Gromov theory of manifold convergence aims to answer the following questions.

Let $\left(\left(M_{i}, g_{i}\right)\right)_{i \geq 1}$ be a sequence of Riemannian manifolds. What does it mean for this sequence to converge to a metric space? Given uniform quantitative bounds on each $\left(M_{i}, g_{i}\right)$, can one deduce the convergence of a subsequence? What are the weakest necessary uniform bounds to derive a (pre-)compactness result?

Convergence results for sequences $\left(\left(M_{i}, g_{i}\right)\right)_{i \geq 1}$ of Riemannian manifolds are first proved in [6] and [12], see also [19] and [11], under the assumption of pointwise bounds on the 
sectional curvature, and are usually refered to as the Cheeger-Gromov theory of manifold convergence.

An important development in the context of this paper are [2] and [8] which obtain pre-compactness results for sequences of Riemannian $n$-manifolds under the assumption of pointwise bounds on the Ricci tensor, $L^{n / 2}$-bounds on the Riemann curvature tensor and that $r_{v o l}\left(M_{i}, 1\right)>0$. Subsequently, pre-compactness results under the assumption of only uniform $L^{p}$-bounds on the Riemann curvature tensor for $p>n / 2$ and a uniform positive lower bound on the volume radius are derived in [3] and [20], see also [28].

While the above results are for manifolds without boundary, convergence results for sequences of Riemannian manifolds with boundary are studied for example in [13], [4], [5], [18] and [16]. Note that these results assume pointwise estimates on the Ricci tensor. We refer the reader to the survey articles [23] and [17].

Compared to the above literature results, in this paper we prove a pre-compactness result for sequences $\left(\left(M_{i}, g_{i}\right)\right)_{i \geq 1}$ of Riemannian 3-manifolds with boundary under the assumption of uniform $L^{2}$-bounds on the Ricci tensor and $L^{4}$-bounds on the second fundamental form of the boundary, and a uniform positive lower bound on the volume radius, see Theorem 5.1 with Corollary 4.14.

The novely in our result is the application of a geometric trace estimate to control the Gauss curvature of the boundary of the manifold in low regularity (see also the next Section 1.2) and a new rigidity argument based on the manifold double. Our presentation follows [20] and [21].

1.2. Boundary harmonic coordinates. Our pre-compactness result for the CheegerGromov theory on manifolds with boundary in low regularity is based on so-called boundary harmonic coordinates. These were already studied in [5] in a higher regularity context. They are defined as follows.

On a Riemannian 3-manifold $(M, g)$, let $\left(x^{1}, x^{2}, x^{3}\right)$ be a local coordinate system near the boundary such that $\left\{x^{3}=0\right\} \subset \partial M$. These coordinates are called boundary harmonic if for $i=1,2,3$ and $A=1,2$

$$
\begin{gathered}
\triangle_{g} x^{i}=0 \text { in }\left\{x^{3}>0\right\} \subset M, \\
\triangle_{g} x^{A}=0 \text { on }\left\{x^{3}=0\right\} \subset \partial M .
\end{gathered}
$$

Here $\not g$ and $\psi_{g}$ denote the induced metric and Laplace-Beltrami operator on $\partial M$, respectively.

Boundary harmonic coordinates are particularly useful for the analysis of regularity of coordinates because the corresponding metric components $g_{i j}$ and $\not_{A B}$ satisfy the elliptic equations

$$
\begin{aligned}
\triangle_{g} g_{i j}+Q_{i j}(g, \partial g) & =-\operatorname{Ric}_{i j} \text { in }\left\{x^{3}>0\right\} \subset M \\
\frac{1}{2} \triangle_{g} \phi_{A B}+\phi_{A B}(\not g, \not \partial g) & =-K g_{A B} \text { on }\left\{x^{3}=0\right\} \subset \partial M,
\end{aligned}
$$


for $i, j=1,2,3$ and $A, B=1,2$. Here the nonlinearities are schematically given by

$$
\left.Q_{i j} \approx g(\partial g)^{2}, \phi_{A B} \approx \not g(\not \partial \not)\right)^{2}
$$

where $\not \partial \in\left\{\partial_{1}, \partial_{2}\right\}$, and $K$ denotes the Gauss curvature of the boundary $\partial M \subset M$, see Lemma 2.15 .

In Section 5, we study a sequence $\left(g_{i}\right)_{i \geq 1}$ of Riemannian metrics in boundary harmonic coordinates which weakly converges to a limit metric $g$ in $H^{2}$. We prove its strong convergence $g_{i} \rightarrow g$ in $H^{2}$ by applying the following general sequence of elliptic estimates for a Riemannian metric in boundary harmonic coordinates, see Section 5.2 for details.

(1) By a geometric trace estimate (see Section 2.4), it holds that the Gauss curvature $K$ is bounded in $H^{-1 / 2}$ on $\partial M$ by an $L^{2}$-bound on Ric and $L^{4}$-bound on $\Theta$. Subsequently, by standard elliptic estimates applied to (1.2) on $\partial M$, the metric components $\oiint_{A B}$ on $\left\{x^{3}=0\right\} \subset \partial M$ are controlled in $H^{3 / 2}$. Then, using that $\left.g_{A B}\right|_{\left\{x^{3}=0\right\}}=\not_{A B}$ on $\partial M$, it follows by standard global elliptic estimates applied to (1.1) in $M$ that the metric components $g_{A B}$ are bounded in $H^{2}$.

(2) In boundary harmonic coordinates, the inverse metric components $g^{33}$ and $g^{3 A}$ also satisfy elliptic equations analogous to (1.1), see Lemma 2.15. Moreover, on the boundary $\left\{x^{3}=0\right\} \subset \partial M$, Neumann data for the components $g^{33}$ and $g^{3 A}$ is explicitly determined by

$$
\begin{aligned}
& N\left(g^{33}\right)=2 \operatorname{tr} \Theta g^{33}, \\
& N\left(g^{3 A}\right)=\operatorname{tr} \Theta g^{3 A}-\frac{1}{2} \frac{1}{\sqrt{g^{33}}} g^{A i} \partial_{i} g^{33},
\end{aligned}
$$

where $N$ denotes the outward-pointing unit normal to $\partial M$, see Lemma 2.17. Consequently, by standard elliptic estimates for the Neumann problem with Neumann data (1.3), it follows that the inverse metric components $g^{33}$ and $g^{3 A}$ are bounded in $H^{2}$ in $M$ by an $L^{2}$-bound on Ric and an $L^{4}$-bound on $\Theta$.

(3) We note that the above $H^{2}$-bounds on the metric components $g_{A B}, g^{33}$ and $g^{3 A}$ imply $H^{2}$-bounds for all metric components $g_{i j}, i, j=1,2,3$. This follows from Kramer's rule and standard product estimates for $H^{2}$-functions.

1.3. Overview of the paper. In Section 2, we introduce notations, function spaces and the Riemannian geometry setting. In Section 3, we state a precise version of our main result. In Section 4, we set up the theory of manifold convergence for manifolds with boundary in low regularity. In Sections 5 and 6, we prove parts (1) and (2) of Theorem 3.1 , respectively. In the appendix, we collect the standard elliptic regularity theory used in Sections 4, 5 and 6.

1.4. Acknowledgements. This work forms part of my Ph.D. thesis. I am grateful to my Ph.D. advisor Jérémie Szeftel for his kind supervision and careful guidance. Furthermore, I would like to thank Lars Andersson for suggesting the idea to use manifold doubling. This work is financially supported by the RDM-IdF. 


\section{Notations, Definitions AND PREREQUisites}

In this section, we introduce notations, definitions and preliminary results that are used in this paper.

2.1. Basic notation. In this work, uppercase Latin indices run trough $A, B, C, D, E, F=$ 1,2 and lowercase Latin indices through $a, b, c, d, i, j=1,2,3$. Greek indices run through $\alpha, \beta, \gamma, \delta, \mu, \nu=0,1,2,3$. We tacitly use the Einstein summation convention. We write $A \lesssim B$ if there exists a universal constant $C>0$ such that $A \leq C B$.

We denote strong and weak convergence by $\rightarrow$ and $\rightarrow$, respectively. We abuse notation by keeping the same index while going to a subsequence of a sequence.

Let the closed upper half-space of $\mathbb{R}^{3}$ be denoted by

$$
\mathbb{H}^{+}:=\left\{x \in \mathbb{R}^{3} \mid x^{3} \geq 0\right\}
$$

and for points $x \in \mathbb{H}^{+}$and real numbers $r>0$ let

$$
\begin{array}{rlrl}
B(x, r) & :=\left\{y \in \mathbb{R}^{3}|| x-y \mid<r\right\}, & S(x, r):=\left\{y \in \mathbb{R}^{3}|| x-y \mid=r\right\}, \\
B^{+}(x, r):=B(x, r) \cap \mathbb{H}^{+}, & S^{+}(x, r):=S(x, r) \cap \mathbb{H}^{+}, \\
\underline{B}^{+}(x, r):=B^{+}(x, r) \cap\left\{x^{3}=0\right\}, & \underline{S}^{+}(x, r):=S^{+}(x, r) \cap\left\{x^{3}=0\right\} .
\end{array}
$$

In given coordinates $\left(x^{1}, x^{2}, x^{3}\right)$, let $\not \partial \in\left\{\partial_{1}, \partial_{2}\right\}$ and $\partial \in\left\{\partial_{1}, \partial_{2}, \partial_{3}\right\}$.

An open subset of $\mathbb{R}^{n}, n \geq 1$, or of $\mathbb{H}^{+}$has smooth boundary if its closure has smooth boundary. A smooth domain is an open subset $\Omega$ of $\mathbb{R}^{n}, n \geq 1$, or $\mathbb{H}^{+}$which is connected and has smooth boundary $\partial \Omega$. Denote by

$$
\underline{\Omega}:=\Omega \cap\left\{x^{3}=0\right\} .
$$

We note that $B^{+}(x, r)$ and $\underline{B}^{+}(x, r)$ have Lipschitz-regular and smooth boundary, respectively.

For $x \in \mathbb{H}^{+}$and two reals $0<r^{\prime}<r$, let $\Omega_{x, r^{\prime}, r} \subset \mathbb{H}^{+}$be a smooth domain such that

$$
\begin{aligned}
& B^{+}\left(x, r^{\prime}\right) \subset \subset \Omega_{x, r^{\prime}, r} \subset \subset B^{+}(x, r), \\
& \underline{B}^{+}\left(x, r^{\prime}\right) \subset \subset \underline{\Omega}_{x, r^{\prime}, r} \subset \subset \underline{B}^{+}(x, r),
\end{aligned}
$$

and define the corresponding smooth cut-off function $\chi_{x, r^{\prime}, r}: \mathbb{H}^{+} \rightarrow[0,1]$ such that

$$
\begin{aligned}
\left.\chi_{x, r^{\prime}, r}\right|_{B^{+}\left(x, r^{\prime}\right)} & \equiv 1, \\
\operatorname{supp} \chi_{x, r^{\prime}, r} & \subset \subset \Omega_{x, r^{\prime}, r} .
\end{aligned}
$$

See also the shaded region in Figure 1. 


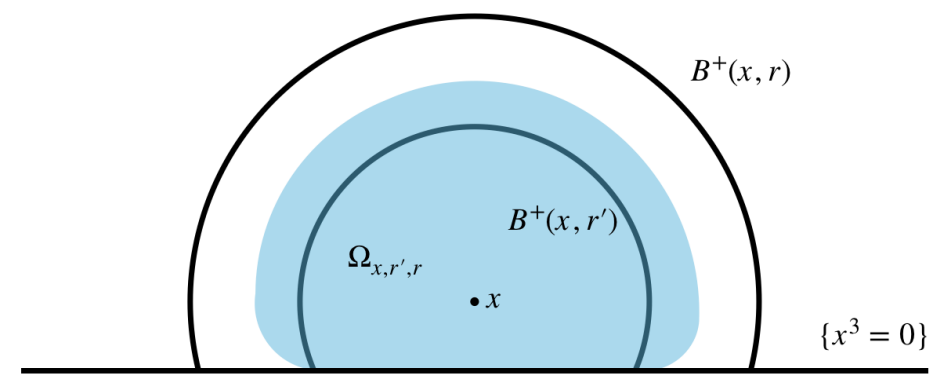

Figure 1. The smooth domain $\Omega_{x, r^{\prime}, r}$ is depicted as the shaded region.

2.2. Function and tensor spaces. In this section, we introduce the function and tensor spaces that are used in this paper. Let $n \geq 1$ be an integer.

\subsubsection{Continuous and Hölder-continuous functions.}

Definition 2.1. Let $m \geq 0$ be an integer and $\alpha \in(0,1]$ a real. Let $\Omega \subset \mathbb{R}^{n}$ be an open bounded set and $f$ a scalar function on $\Omega$. Define the Hölder semi-norm by

$$
[f]_{C^{0, \alpha}(\bar{\Omega})}:=\sup _{x \neq y \in \bar{\Omega}} \frac{|f(x)-f(y)|}{|x-y|^{\alpha}}
$$

and let the norm

$$
\|f\|_{C^{m, \alpha}(\bar{\Omega})}:=\|f\|_{C^{m}(\bar{\Omega})}+\max _{|\beta|=m}\left[\partial^{\beta} f\right]_{C^{0, \alpha}(\bar{\Omega})}
$$

where

$$
\|f\|_{C^{m}(\bar{\Omega})}:=\max _{|\beta| \leq m} \sup _{x \in \bar{\Omega}}\left|\partial^{\beta} f\right| .
$$

Here $\beta \in \mathbb{N}^{n}$ is a multi-index and

$$
|\beta|:=\left|\beta_{1}\right|+\cdots+\left|\beta_{n}\right|, \quad \partial^{\beta}:=\partial_{1}^{\beta_{1}} \ldots \partial_{n}^{\beta_{n}} .
$$

Let $C^{m}(\bar{\Omega})$ be the space of m-times continuously differentiable functions on $\bar{\Omega}$ equipped with the norm $\|\cdot\|_{C^{m}(\bar{\Omega})}$. Let the Hölder spaces $C^{m, \alpha}(\bar{\Omega})$ be defined by

$$
C^{m, \alpha}(\bar{\Omega}):=\left\{f \in C^{m}(\bar{\Omega}):\|f\|_{C^{m, \alpha}(\bar{\Omega})}<\infty\right\} .
$$

2.2.2. Fractional Sobolev spaces. In this section we define the fractional Sobolev spaces $W^{s, p}\left(\mathbb{R}^{n}\right)$ for reals $s \in \mathbb{R}$ and $1<p<\infty$, and summarise basic properties. For more details, see for example [24] and [1].

Definition 2.2 (Fractional Sobolev spaces). Let $\Omega \subset \mathbb{R}^{n}$ be an open set, and let $s \in \mathbb{R}$ and $1<p<\infty$ be two reals. The function space $W^{s, p}(\Omega)$ is defined as 
- If $s \geq 0$ integer,

$$
W^{s, p}(\Omega):=\left\{f \in L^{p}(\Omega):\|f\|_{W^{s, p}(\Omega)}^{p}<\infty\right\},
$$

where

$$
\|f\|_{W^{s, p}(\Omega)}^{p}:=\sum_{|\beta| \leq s}\left\|\partial^{\beta} f\right\|_{L^{p}(\Omega)}^{p} .
$$

- If $s \in(0,1)$,

$$
W^{s, p}(\Omega):=\left\{f \in L^{p}(\Omega):\|f\|_{W^{s, p}(\Omega)}^{p}<\infty\right\},
$$

where

$$
\|f\|_{W^{s, p}(\Omega)}^{p}:=\|f\|_{L^{p}(\Omega)}^{p}+\int_{\Omega} \int_{\Omega} \frac{|f(x)-f(y)|^{p}}{|x-y|^{n+s p}} d x d y .
$$

- If $s=k+\theta$ with $k \geq 0$ integer, $\theta \in(0,1)$,

$$
W^{s, p}(\Omega):=\left\{f \in L^{p}(\Omega):\|f\|_{W^{s, p}(\Omega)}<\infty\right\} .
$$

where

$$
\|f\|_{W^{s, p}(\Omega)}:=\|f\|_{W^{k, p}(\Omega)}+\sum_{|\alpha|=k}\left\|\partial^{\alpha} f\right\|_{W^{\theta, p}(\Omega)} .
$$

For $s<0$, let $W^{s, p}(\Omega)$ be the dual space to the closure of compactly supported smooth functions with respect to the topology of $W^{-s, \frac{p}{p-1}}(\Omega)$, denoted by $W_{0}^{-s, \frac{p}{p-1}}(\Omega)$.

For $s>0$ not integer, $W^{s, p}$ is called Sobolev-Slobodeckij space in the literature.

Remark 2.3. For $s \in \mathbb{R}$ and $p=2$, we have the identification

$$
W^{s, p}\left(\mathbb{R}^{n}\right)=H^{s}\left(\mathbb{R}^{n}\right),
$$

where $H^{s}\left(\mathbb{R}^{n}\right)$ is the Sobolev space defined

- for $s<0$ by

$H^{s}\left(\mathbb{R}^{n}\right):=\operatorname{closure}\left(\left\{f \in \mathcal{S}^{\prime}\left(\mathbb{R}^{n}\right): \widehat{f} \in L_{\text {loc }}^{1}\left(\mathbb{R}^{n}\right),\left\|\left(1+|\xi|^{2}\right)^{s / 2} \widehat{f}\right\|_{L^{2}\left(\mathbb{R}^{n}\right)}<\infty\right\}\right)$.

- for $s \geq 0$ by

$$
H^{s}\left(\mathbb{R}^{n}\right):=\operatorname{closure}\left(\left\{f \in \mathcal{S}\left(\mathbb{R}^{n}\right):\left\|\left(1+|\xi|^{2}\right)^{s / 2} \widehat{f}\right\|_{L^{2}\left(\mathbb{R}^{n}\right)}<\infty\right\}\right) .
$$

In the above, the closure is taken with respect to the indicated norm, and the space $\mathcal{S}\left(\mathbb{R}^{n}\right)$ denotes the space of Schwartz functions on $\mathbb{R}^{n}$ and $\mathcal{S}^{\prime}\left(\mathbb{R}^{n}\right)$ its dual space, also called the 
space of tempered distributions. Moreover, the Fourier transform $\widehat{\cdot}: \mathcal{S}\left(\mathbb{R}^{n}\right) \rightarrow \mathcal{S}\left(\mathbb{R}^{n}\right)$ and its inverse $^{\vee}: \mathcal{S}\left(\mathbb{R}^{n}\right) \rightarrow \mathcal{S}\left(\mathbb{R}^{n}\right)$ are defined for $f_{1}, f_{2} \in \mathcal{S}\left(\mathbb{R}^{n}\right)$ by

$$
\begin{aligned}
& \widehat{f}_{1}(\xi):=\int_{\mathbb{R}^{n}} e^{-i 2 \pi x \xi} f_{1}(x) d x, \\
& \vee \\
& f_{2}(x):=\frac{1}{(2 \pi)^{n}} \int_{\mathbb{R}^{n}} e^{i 2 \pi x \xi} f_{2}(\xi) d \xi .
\end{aligned}
$$

We recall that $\widehat{\cdot}$ and $\stackrel{\vee}{\cdot}$ extend to mappings $\widehat{\cdot}: \mathcal{S}^{\prime} \rightarrow \mathcal{S}^{\prime}$ and $\stackrel{\vee}{\cdot}: \mathcal{S}^{\prime} \rightarrow \mathcal{S}^{\prime}$, see [22].

For an open set $\Omega \subset \mathbb{R}^{n}$ and $s \in \mathbb{R}$, we denote on $H^{s}(\Omega)=W^{s, 2}(\Omega)$.

The following lemma follows from more general Sobolev embeddings for $W^{s, p}$ spaces, see for example [24] and [1] for details and proofs.

Lemma 2.4 (Sobolev embeddings). Let $\Omega^{2} \subset \mathbb{R}^{2}$ and $\Omega^{3} \subset \mathbb{R}^{3}$ be smooth domains. Then, the following are continuous embeddings,

$$
\begin{aligned}
& H^{1 / 2}\left(\Omega^{2}\right) \hookrightarrow L^{4}\left(\Omega^{2}\right), \\
& L^{2}\left(\Omega^{2}\right) \hookrightarrow H^{-1 / 2}\left(\Omega^{2}\right) .
\end{aligned}
$$

If, furthermore, $\Omega^{2}$ and $\Omega^{3}$ are bounded, then the following continuous embeddings are also compact,

$$
\begin{aligned}
& H^{2}\left(\Omega^{3}\right) \hookrightarrow C^{0, \alpha}\left(\overline{\Omega^{3}}\right) \text { for } \alpha \in(0,1 / 2), \\
& H^{2}\left(\Omega^{3}\right) \hookrightarrow W^{1,4}\left(\Omega^{3}\right), \\
& H^{2}\left(\Omega^{3}\right) \hookrightarrow H^{1}\left(\Omega^{3}\right), \\
& H^{3 / 2}\left(\Omega^{2}\right) \hookrightarrow H^{1 / 2}\left(\Omega^{2}\right) .
\end{aligned}
$$

The following are standard product estimates, we refer the reader to Section 13.3 in [26] and [15] for more details and proofs.

Lemma 2.5 (Product estimates). Let $\Omega^{2} \subset \mathbb{R}^{2}$ be a smooth domain, and let $u$ and $v$ be functions on $\Omega^{2}$. Then,

$$
\|u v\|_{H^{1 / 2}\left(\Omega^{2}\right)} \lesssim\|u\|_{H^{5 / 4}\left(\Omega^{2}\right)}\|v\|_{H^{1 / 2}\left(\Omega^{2}\right)} .
$$

Moreover, for every integer $m \geq 0$, there is a constant $C_{m}>0$ such that

$$
\begin{gathered}
\|u v\|_{H^{m+3 / 2}\left(\Omega^{2}\right)} \lesssim\|u\|_{H^{m+3 / 2}\left(\Omega^{2}\right)}\|v\|_{H^{3 / 2}\left(\Omega^{2}\right)}+\|u\|_{H^{3 / 2}\left(\Omega^{2}\right)}\|v\|_{H^{m+3 / 2}\left(\Omega^{2}\right)} \\
+C_{m}\left(\|u\|_{H^{3 / 2}\left(\Omega^{2}\right)}+\|v\|_{H^{3 / 2}\left(\Omega^{2}\right)}\right) .
\end{gathered}
$$

Let $\Omega^{3} \subset \mathbb{R}^{3}$ be a smooth domain, and let $u$ and $v$ be functions on $\Omega^{3}$. Then for every integer $m \geq 0$ there is a constant $C_{m}>0$ such that

$$
\begin{gathered}
\|u v\|_{H^{m}\left(\Omega^{3}\right)} \lesssim\|u\|_{H^{m}\left(\Omega^{3}\right)}\|v\|_{H^{2}\left(\Omega^{3}\right)}+\|u\|_{H^{2}\left(\Omega^{3}\right)}\|v\|_{H^{m}\left(\Omega^{3}\right)} \\
+C_{m}\left(\|u\|_{H^{2}\left(\Omega^{3}\right)}+\|v\|_{H^{2}\left(\Omega^{3}\right)}\right) .
\end{gathered}
$$


We define now the trace operator for continuous functions.

Definition 2.6 (Trace operator for continuous functions). Let $n \geq 1$ be an integer and let $\Omega \subset \mathbb{R}^{n}$ be a smooth domain. Let $f$ be a continuous scalar function on $\bar{\Omega}$. Denote the restriction of $f$ to $\partial \Omega$ by

$$
\tau(f):=\left.f\right|_{\partial \Omega} .
$$

The trace operator $\tau$ extends to Sobolev spaces as follows.

Lemma 2.7 (Trace operator for $W^{k, p}$-functions). Let $n \geq 1$ and $k \geq 1$ be integers and $1<p<\infty$ a real. Let $\Omega \subset \mathbb{R}^{n}$ be a smooth domain. Then the trace operator $\tau$ extends to a bounded linear operator between the following function spaces,

$$
W^{k, p}(\Omega) \rightarrow W^{k-1 / p, p}(\partial \Omega) .
$$

Remark 2.8. We cannot apply Lemmas 2.4, 2.5 and 2.7 directly to the sets $B^{+}(x, r)$ because they are not smooth domains. Therefore, whenever these lemmas are invoked in Sections 4, 5 and 6, we tacitly apply the lemmas to the smooth domain $\Omega_{x, r^{\prime}, r}$ between $B^{+}\left(x, r^{\prime}\right)$ and $B^{+}(x, r)$, see its definition in (2.2), so that the estimates hold on $B^{+}\left(x, r^{\prime}\right)$ for a slightly smaller $r^{\prime}<r$.

In Section 2.4, we work with the space $H^{-1 / 2}\left(\mathbb{R}^{2}\right)$. To ease the presentation, we now introduce a Fourier operator $\langle\not \partial\rangle^{-1}$ and summarise its basic properties in Lemma 2.10.

Definition 2.9. Let $f \in \mathcal{S}\left(\mathbb{R}^{3}\right)$ be a scalar function. Define

$$
\langle\not \supset\rangle^{-1} f\left(\cdot, x^{3}\right):=\left(\left(1+\left(\xi^{1}\right)^{2}+\left(\xi^{2}\right)^{2}\right)^{-1 / 2} \widehat{f}\left(\xi^{1}, \xi^{2}, x^{3}\right)\right)^{\vee},
$$

where the Fourier transform and its inverse are taken with the respect the variables $x^{1}, x^{2}$ only.

The proof of the next lemma is left to the reader.

Lemma 2.10 (Properties of $\langle\not \partial\rangle^{-1}$ ). The following holds.

- The operator $\langle\not\rangle^{-1}$ extends to a mapping $L^{2}\left(\mathbb{R}^{3}\right) \rightarrow L^{2}\left(\mathbb{R}^{3}\right)$, and for two functions $f, f^{\prime} \in L^{2}\left(\mathbb{R}^{3}\right)$, we have

$$
\int_{\mathbb{R}^{3}} f\left(\langle\not \partial\rangle^{-1} f^{\prime}\right)=\int_{\mathbb{R}^{3}}\left(\langle\not \partial\rangle^{-1} f\right) f^{\prime} .
$$

- Let $f \in \mathcal{S}\left(\mathbb{R}^{3}\right)$ be a scalar function and $s \in \mathbb{R}$,

$$
\left\|\langle\not \partial\rangle^{-1} f\left(\cdot, x^{3}\right)\right\|_{H^{s+1}\left(\mathbb{R}^{2}\right)}=\left\|f\left(\cdot, x^{3}\right)\right\|_{H^{s}\left(\mathbb{R}^{2}\right)} .
$$

- Let $f \in \mathcal{S}\left(\mathbb{R}^{3}\right)$ be a scalar function, then

$$
\begin{aligned}
\left\|f\left(\cdot, x^{3}\right)\right\|_{H^{-1 / 2}\left(\mathbb{R}^{2}\right)}^{2} & :=\left\|\left(1+|\cdot|^{2}\right)^{-1 / 4} \widehat{f}\left(\cdot, x^{3}\right)\right\|_{L^{2}\left(\mathbb{R}^{2}\right)}^{2} \\
& =\int_{\mathbb{R}^{2}} f\left(x^{1}, x^{2}, x^{3}\right)\langle\not\rangle^{-1} f\left(x^{1}, x^{2}, x^{3}\right) d x^{1} d x^{2} .
\end{aligned}
$$


- Let $f \in \mathcal{S}\left(\mathbb{R}^{3}\right)$ be a scalar function, then

$$
\left[\partial_{x^{3}},\langle\not \supset\rangle^{-1}\right] f=0 .
$$

2.2.3. Tensor spaces.

Definition 2.11 (Tensor spaces). Let $n \geq 1$ be an integer. Let $\Omega \subset \mathbb{R}^{n}$ be an open set and let $T$ be a tensor on $\Omega$. For reals $s \in \mathbb{R}$ and $1<p<\infty$, and integers $k \geq 0$, we let

$$
\mathcal{W}^{s, p}(\Omega), \mathcal{H}^{s}(\Omega), \mathcal{L}^{p}(\Omega) \text { and } \mathcal{C}^{k}(\Omega)
$$

denote the spaces of tensors whose coordinate components are respectively in

$$
W^{s, p}(\Omega), H^{s}(\Omega), L^{p}(\Omega) \text { and } C^{k}(\Omega),
$$

equipped with the standard norm, that is, for example, for a $(l, m)$-tensor $T$ on $\Omega$,

$$
\|T\|_{\mathcal{W}^{s, p}(\Omega)}:=\sum_{i_{1}, \ldots i_{l}=1}^{n} \sum_{j_{1} \ldots j_{m}=1}^{n}\left\|T_{j_{1} \ldots j_{m}}^{i_{1} \ldots i_{l}}\right\|_{W^{s, p}(\Omega)}
$$

where $T_{j_{1} \ldots j_{m}}^{i_{1} \ldots i_{l}}$ denotes the coordinate components.

\subsection{Riemannian geometry and boundary harmonic coordinates.}

Definition 2.12 (Volume radius at scale $r$ ). Let $(M, g)$ be a Riemannian 3-manifold with boundary. For a real $r>0$ and a point $p \in M$, the volume radius at scale $r$ at $p$ is defined as

$$
r_{v o l}(r, p):=\inf _{r^{\prime}<r} \frac{\operatorname{vol}_{g}\left(B_{g}\left(p, r^{\prime}\right)\right)}{\frac{4 \pi}{3}\left(r^{\prime}\right)^{3}},
$$

where $B_{g}\left(p, r^{\prime}\right)$ denotes the geodesic ball of radius $r^{\prime}$ centered at $p$. The volume radius of $(M, g)$ at scale $r$ is defined as

$$
r_{v o l}(M, r):=\inf _{p \in M} r_{v o l}(r, p) .
$$

Definition 2.13 (Boundary harmonic coordinates). Let $(M, g)$ be a smooth Riemannian 3-manifold with boundary. For a point $x \in \mathbb{H}^{+}$and a real $r>0$, let $\varphi: B^{+}(x, r) \rightarrow U \subset M$ be a chart of $M$ such that $\left\{x^{3}=0\right\} \subset \partial M$. The coordinates $\left(x^{1}, x^{2}, x^{3}\right)$ are called boundary harmonic if

$$
\begin{gathered}
\triangle_{g} x^{j}=0 \text { on } B^{+}(x, r) \text { for } j=1,2,3, \\
\triangle_{g} x^{A}=0 \text { on } \underline{B}^{+}(x, r) \text { for } A=1,2 .
\end{gathered}
$$

Here $\Psi_{g}$ denotes Laplace-Beltrami operator of the induced metric $\not g$ on $\underline{B}^{+}(x, r)$. In this case, we also call the chart $\varphi: B^{+}(x, r) \rightarrow U \subset M$ boundary harmonic.

Remark 2.14. In the above definition, as in the rest of this paper, we abuse notation by not explicitly denoting the pullback of $g$ by $\varphi$. For example, we write $\triangle_{g} x^{j}$ on $B^{+}(x, r)$ instead of $\triangle_{\varphi^{*} g} x^{j}$ on $B^{+}(x, r)$. 
The proof of the following properties of boundary harmonic coordinates is left to the reader, see also Lemma 11.2.6 in [20] for the case of manifolds without boundary.

Lemma 2.15. Let $g$ be a Riemannian metric in boundary harmonic coordinates $\left(x^{1}, x^{2}, x^{3}\right)$ on $B^{+}(x, r)$. Then the following holds for $i, j=1,2,3$ and $A, B=1,2$.

- The coordinate components $g_{i j}$ satisfy on $B^{+}(x, r)$

$$
\frac{1}{2} \triangle_{g} g_{i j}+Q_{i j}=-\mathrm{Ric}_{i j}
$$

where $Q_{i j}(g, \partial g):=-g\left(\nabla g_{i k}, \nabla g^{k l}\right) g_{l j}+g\left(\operatorname{Hess} x^{k}\right.$, Hess $\left.x^{l}\right) g_{i k} g_{l j}$ and Ric is the Ricci tensor of $g$.

- The components $g^{i j}$ of the inverse metric satisfy on $B^{+}(x, r)$

$$
\frac{1}{2} \triangle_{g} g^{i j}-Q^{i j}=\operatorname{Ric}^{i j}
$$

where $Q^{i j}:=g\left(\operatorname{Hess} x^{i}, \operatorname{Hess} x^{j}\right)$, and $\operatorname{Ric}^{i j}=g^{i l} g^{j m} \operatorname{Ric}_{l m}$.

- The coordinate components of the induced metric $\oiint_{A B}$ satisfy on $\underline{B}^{+}(x, r)$

$$
\frac{1}{2} \phi_{g} \phi_{A B}+\phi_{A B}=-K \phi_{A B},
$$

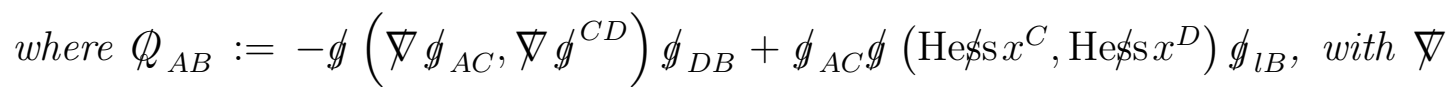
the induced covariant derivative on $\underline{B}^{+}(x, r)$, and $K$ denotes the Gauss curvature of $\underline{B}^{+}(x, r)$.

- The Laplace-Beltrami operators on $B^{+}(x, r)$ and $\underline{B}^{+}(x, r)$ are respectively given by

$$
\triangle_{g} u=g^{i j} \partial_{i} \partial_{j} u, \triangle_{g} u=\phi^{A B} \not_{A} \partial_{B} u \text {. }
$$

The quadratic non-linearities $\phi_{A B} \approx \not \partial \not \partial \not \partial$ and $Q_{i j}, Q^{i j} \approx \partial g \partial g$ satisfy the following properties. The proof follows by standard Sobolev embeddings and product estimates (see Lemmas 2.4 and 2.5), and is left to the reader.

Lemma 2.16. Let $\Omega^{2} \subset \mathbb{R}^{2}$ and $\Omega^{3} \subset \mathbb{R}^{3}$ be bounded smooth domains. Then the following holds.

- Let $\left(\oiint_{n}\right)_{n \geq 0}$ and $\not$ be smooth Riemannian metrics on $\Omega^{2}$. If

$$
\not \phi_{n} \rightarrow \not g \text { in } H^{3 / 2}\left(\Omega^{2}\right) \text { as } n \rightarrow \infty,
$$

then

$$
\left\|\phi_{A B}\left(\not \phi_{n}\right)-\phi_{A B}(\not)\right\|_{H^{-1 / 2}\left(\Omega^{2}\right)} \rightarrow 0 \text { as } n \rightarrow \infty .
$$

- Let $\left(g_{n}\right)_{n \geq 0}$ and $g$ be smooth Riemannian metrics on $\Omega^{3}$. If

$$
g_{n} \rightarrow g \text { in } H^{2}\left(\Omega^{3}\right) \text { as } n \rightarrow \infty,
$$

then

$$
\left\|Q_{A B}\left(g_{n}\right)-Q_{A B}(g)\right\|_{L^{2}\left(\Omega^{3}\right)} \rightarrow 0 \text { as } n \rightarrow \infty .
$$


- There is $\varepsilon>0$ small such that if

$$
\|\not h-e\|_{\mathcal{H}^{3 / 2}\left(\Omega^{2}\right)}<\varepsilon,
$$

then for every integer $m>0$ there is a constant $C_{m}>0$ such that

$$
\begin{gathered}
\left\|\not{ }^{m-1} \phi_{A B}\right\|_{H^{1 / 2}\left(\Omega^{2}\right)} \lesssim\|\not g-e\|_{\mathcal{H}^{3 / 2}\left(\Omega^{2}\right)}\left\|\not \partial^{m+1} \not\right\|_{\mathcal{H}^{1 / 2}\left(\Omega^{2}\right)}+\|\not\|_{\mathcal{H}^{m+1 / 2}\left(\Omega^{2}\right)} \\
+C_{m}\|\not g-e\|_{\mathcal{H}^{3 / 2}\left(\Omega^{2}\right)} .
\end{gathered}
$$

- There is $\varepsilon>0$ small such that if

$$
\|g-e\|_{\mathcal{H}^{2}\left(\Omega^{3}\right)}<\varepsilon,
$$

then for every integer $m>0$ there is a constant $C_{m}>0$ such that

$$
\begin{aligned}
& \left\|\partial^{m} Q_{A B}\right\|_{L^{2}\left(\Omega^{3}\right)} \lesssim\|g-e\|_{\mathcal{H}^{2}\left(\Omega^{3}\right)}\left\|\partial^{m+2} g\right\|_{\mathcal{L}^{2}\left(\Omega^{3}\right)}+\|g\|_{\mathcal{H}^{m+1}\left(\Omega^{3}\right)} \\
& +C_{m}\|g-e\|_{\mathcal{H}^{2}\left(\Omega^{3}\right)} \text {. }
\end{aligned}
$$

In boundary harmonic coordinates $\left(x^{1}, x^{2}, x^{3}\right)$ on $B^{+}(x, r)$, we can express $g$ as

$$
g=a^{2}\left(d x^{3}\right)^{2}+\not g_{A B}\left(\beta^{A} d x^{3}+d x^{A}\right)\left(\beta^{B} d x^{3}+d x^{B}\right) \text {. }
$$

with

- the lapse function $a>0$,

- the induced Riemannian metric $\not f$ on level sets $\left\{x^{3}=\right.$ const. $\}$,

- the shift vector $\beta$, tangent to $\left\{x^{3}=\right.$ const. $\}$.

The outward-pointing ${ }^{3}$ unit normal to the level sets $\left\{x^{3}=\right.$ const. $\}$ is given by

$$
N:=-\frac{1}{a} \partial_{3}+\frac{1}{a} \beta^{A} \partial_{A}=-a \nabla x^{3} .
$$

It holds that $\operatorname{det} g=a^{2} \operatorname{det} \not g$ and the components $g^{i j}$ of the metric inverse are given by

$$
g^{A B}=\phi^{A B}+\frac{\beta^{A} \beta^{B}}{a^{2}}, g^{A 3}=-\frac{\beta^{A}}{a^{2}}, g^{33}=\frac{1}{a^{2}} .
$$

The second fundamental form ${ }^{4}$ on $\underline{B}^{+}(x, r)$ is given in general coordinates by

$$
\Theta_{A B}=\frac{1}{2 a} \partial_{3}\left(g_{A B}\right)-\frac{1}{2 a}\left(\mathscr{A}_{\beta} \not g\right)_{A B},
$$

where $\mathcal{A}$ denotes the induced Lie derivative on $\underline{B}^{+}(x, r)$.

Lemma 2.17. Let $g$ be a Riemannian metric in boundary harmonic coordinates on $B^{+}(x, r)$. Let $N$ and $\Theta$ denote the outward-pointing unit normal and the second fundamental form of $\underline{B}^{+}(x, r)$, respectively. Then it holds that on $\underline{B}^{+}(x, r)$

$$
\begin{aligned}
& N\left(g^{33}\right)=2 \operatorname{tr} \Theta g^{33} \\
& N\left(g^{3 A}\right)=\operatorname{tr} \Theta g^{3 A}-\frac{1}{2} \frac{1}{\sqrt{g^{33}}} g^{A m} \partial_{m} g^{33} .
\end{aligned}
$$

\footnotetext{
${ }^{3}$ The outward-pointing unit normal has by definition negative $\partial_{x^{3}}$-component.

${ }^{4}$ Here we use the sign convention $\Theta(X, Y):=-g\left(X, \nabla_{Y} N\right)$.
} 
Proof. In general, for smooth functions $f$,

$$
\triangle_{g} f=N(N(f))-\operatorname{tr} \Theta N(f)+\not_{g} f+a^{-1} g(\not \nabla a, \not \nabla f) .
$$

By assumption we have for $j=1,2,3$ that $\triangle x^{j}=0$ and $\triangle 4 x^{j}=0$. Hence

$$
0=\triangle x^{j}=N\left(N\left(x^{j}\right)\right)-\operatorname{tr} \Theta N\left(x^{j}\right)+a^{-1} g\left(\not \nabla a, \not \nabla x^{j}\right) .
$$

By (2.6) and (2.7) it follows for $A=1,2$ that

$$
\begin{aligned}
N\left(N\left(x^{3}\right)\right) & =-N\left(\sqrt{g^{33}}\right) \\
& =-\frac{1}{2} a N\left(g^{33}\right), \\
N\left(N\left(x^{A}\right)\right) & =-N\left(a g^{3 A}\right) \\
& =-N(a) g^{3 A}-a N\left(g^{3 A}\right) \\
& =-N(a) g\left(\nabla x^{3}, \nabla x^{A}\right)-a N\left(g^{3 A}\right) \\
& =a^{-1} g\left(\nabla x^{A}, N\right) N(a)-a N\left(g^{3 A}\right),
\end{aligned}
$$

and moreover

$$
\operatorname{tr} \Theta N\left(x^{j}\right)=-\operatorname{tr} \Theta a g^{3 j}
$$

By (2.10), (2.11), (2.9) and using that $\not x^{3}=0$, we get

$$
\begin{aligned}
N\left(g^{33}\right) & =2 \operatorname{tr} \Theta g^{33} \\
N\left(g^{3 A}\right) & =a^{-2}\left(g\left(\nabla x^{A}, N\right) N(a)+g\left(\not \nabla x^{A}, \not \nabla a\right)\right)+\operatorname{tr} \Theta g^{3 A} \\
& =a^{-2}\left(\nabla x^{A}\right)(a)+\operatorname{tr} \Theta g^{3 A} \\
& =-\frac{1}{2 \sqrt{g^{33}}}\left(\nabla x^{A}\right)\left(g^{33}\right)+\operatorname{tr} \Theta g^{3 A} .
\end{aligned}
$$

This finishes the proof of Lemma 2.17.

The next lemma shows that bounds on the metric components $g^{33}, g^{3 A}, g_{A B}$ imply bounds for all metric components $g_{i j}$.

Lemma 2.18 (Control of all metric components). Let $\Omega \subset \mathbb{R}^{3}$ be a smooth domain and let $g$ be a smooth Riemannian metric on $\Omega$. There is $\varepsilon>0$ such that if

$$
\|g-e\|_{\mathcal{H}^{2}(\Omega)}<\varepsilon,
$$

then for every integer $m \geq 0$, there is a constant $C_{m}>0$ such that

$$
\|g\|_{\mathcal{H}^{m}(\Omega)} \lesssim \sum_{A, B=1,2}\left(\left\|g_{A B}\right\|_{\mathcal{H}^{m}(\Omega)}+\left\|g^{33}\right\|_{\mathcal{H}^{m}(\Omega)}+\left\|g^{3 A}\right\|_{\mathcal{H}^{m}(\Omega)}\right)+C_{m}\|g-e\|_{\mathcal{H}^{2}(\Omega)} .
$$


Proof. It suffices to control the components $g_{3 A}$ and $g_{33}$. By (2.5), (2.7), and the general property $g_{3 k} g^{k 3}=1$,

$$
g_{3 A}=g_{A C} \frac{g^{3 C}}{g^{33}}, g_{33}=\frac{1}{g^{33}}\left(1-g_{3 A} g^{3 A}\right) .
$$

From this, the product estimates of Lemma 2.5 imply (2.12). Details are left to the reader.

2.4. The trace estimate for the Gauss curvature $K$. Let $g$ be a Riemannian metric in boundary harmonic coordinates on $B^{+}(x, r)$ such that

$$
\|g-e\|_{\mathcal{H}^{2}\left(B^{+}(x, r)\right)}<\varepsilon
$$

for a small constant $\varepsilon>0$.

In this section, we prove that if $\varepsilon>0$ is sufficiently small, then for all $0<r^{\prime}<r$ it holds that

$$
\|K\|_{H^{-1 / 2}\left(\underline{B}^{+}\left(x, r^{\prime}\right)\right)} \leq C_{r^{\prime}, r}\left(\|\operatorname{Ric}\|_{L^{2}\left(B^{+}(x, r)\right)}+\|\Theta\|_{L^{4}\left(\underline{B}^{+}(x, r)\right)}\right) .
$$

We remark that such a trace estimate was already proved in [14] in Besov spaces, see also [25]. For the convenience of the reader, we give here a proof of (2.13) without Besov spaces.

By the twice contracted Gauss equation

$$
2 K=(\operatorname{tr} \Theta)^{2}-|\Theta|^{2}+\mathrm{R}_{\text {scal }}-2 \operatorname{Ric}(N, N),
$$

and Lemmas 2.4 and 2.7 it follows that for $\varepsilon>0$ sufficiently small,

$$
\|K\|_{H^{-1 / 2}\left(\underline{B}^{+}\left(x, r^{\prime}\right)\right)} \lesssim\left\|\mathrm{R}_{\text {scal }}-2 \operatorname{Ric}(N, N)\right\|_{H^{-1 / 2}\left(\underline{B}^{+}\left(x, r^{\prime}\right)\right)}+\|\Theta\|_{L^{4}\left(\underline{B}^{+}(x, r)\right)} .
$$

Therefore, the proof of (2.13) follows from the next proposition.

Proposition 2.19 (Trace estimate). Let g be a Riemannian metric in boundary harmonic coordinates on $B^{+}(x, r)$. There is an $\varepsilon>0$ small such that if

$$
\|g-e\|_{\mathcal{H}^{2}\left(B^{+}(x, r)\right)}<\varepsilon
$$

then it holds that for all $0<r^{\prime}<r$,

$$
\left\|\mathrm{R}_{\text {scal }}-2 \operatorname{Ric}(N, N)\right\|_{H^{-1 / 2}\left(\underline{B}^{+}\left(x, r^{\prime}\right)\right)} \leq C_{r^{\prime}, r}\|\operatorname{Ric}\|_{L^{2}\left(B^{+}(x, r)\right)} .
$$

In the rest of this section, we prove Proposition 2.19. The proof of this trace estimate is based on the identity

$$
\begin{aligned}
& N\left(\mathrm{R}_{\text {scal }}-2 \operatorname{Ric}_{N N}\right) \\
= & \nabla_{N} \mathrm{R}_{\text {scal }}-2 \nabla_{N} \operatorname{Ric}_{N N}+4 \operatorname{Ric}_{A N}\left(\nabla_{N} N\right)^{A} \\
= & 2 \nabla^{A} \operatorname{Ric}_{A N}+4 \operatorname{Ric}_{A N}\left(\nabla_{N} N\right)^{A} \\
= & 2 \operatorname{dif}(\text { Ric. } N)-2 \operatorname{tr} \Theta \operatorname{Ric}_{N N}+2 \Theta^{A B} \operatorname{Ric}_{A B}+4 \operatorname{Ric}_{A N}\left(\nabla_{N} N\right)^{A} .
\end{aligned}
$$


We note that (2.14) follows from the twice contracted Bianchi identity

$$
\nabla^{l} \operatorname{Ric}_{l j}=\frac{1}{2} \nabla_{l} \mathrm{R}_{s c a l}
$$

Let $\chi:=\chi_{x, r^{\prime}, r}$ be a smooth cut-off function from $B^{+}\left(x, r^{\prime}\right)$ to $B^{+}(x, r)$ as defined in (2.4). By Lemma 2.10 and the fundamental theorem of calculus,

$$
\begin{aligned}
& \left\|\mathrm{R}_{\text {scal }}-2 \mathrm{Ric}_{N N}\right\|_{H^{-1 / 2}\left(\underline{B}^{+}\left(x, r^{\prime}\right)\right)}^{2} \\
\leq & \left\|\chi^{2}\left(\mathrm{R}_{\text {scal }}-2 \operatorname{Ric}_{N N}\right)\right\|_{H^{-1 / 2}\left(\mathbb{R}^{2}\right)}^{2} \\
= & \int_{\left\{x^{3}=0\right\}} \chi^{2}\left(\mathrm{R}_{\text {scal }}-2 \operatorname{Ric}_{N N}\right)\langle\not\rangle^{-1}\left(\chi^{2}\left(\mathrm{R}_{\text {scal }}-2 \operatorname{Ric}_{N N}\right)\right) d x^{1} d x^{2} \\
= & \int_{0}^{r} \partial_{3}\left(\int_{\mathbb{R}^{2}} \chi^{2}\left(\mathrm{R}_{\text {scal }}-2 \operatorname{Ric}_{N N}\right)\langle\not \partial\rangle^{-1}\left(\chi^{2}\left(\mathrm{R}_{\text {scal }}-2 \operatorname{Ric}_{N N}\right)\right) d x^{1} d x^{2}\right) d x^{3} \\
= & 2 \int_{0}^{r} \int_{\mathbb{R}^{2}} \chi^{2}\left(\mathrm{R}_{\text {scal }}-2 \operatorname{Ric}_{N N}\right)\langle\not \partial\rangle^{-1}\left(\partial_{3}\left(\chi^{2}\left(\mathrm{R}_{\text {scal }}-2 \operatorname{Ric}_{N N}\right)\right)\right),
\end{aligned}
$$

Expressing $\partial_{3}=a N+\beta$, see (2.6), we get

$$
\begin{aligned}
& \left\|\mathrm{R}_{\text {scal }}-2 \operatorname{Ric}_{N N}\right\|_{H^{-1 / 2}\left(\underline{B}^{+}\left(x, r^{\prime}\right)\right)}^{2} \\
& \leq \int_{0}^{r} \int_{\mathbb{R}^{2}} \chi^{2}\left(\mathrm{R}_{\text {scal }}-2 \operatorname{Ric}_{N N}\right)\langle\not\rangle^{-1}\left(\chi^{2} a N\left(\mathrm{R}_{\text {scal }}-2 \operatorname{Ric}_{N N}\right)\right) \\
& \quad+\int_{0}^{r} \int_{\mathbb{R}^{2}} \chi^{2}\left(\mathrm{R}_{\text {scal }}-2 \operatorname{Ric}_{N N}\right)\langle\not \partial\rangle^{-1}\left(\chi^{2} \beta\left(\mathrm{R}_{\text {scal }}-2 \operatorname{Ric}_{N N}\right)\right) \\
& \quad+\int_{0}^{r} \int_{\mathbb{R}^{2}} \chi^{2}\left(\mathrm{R}_{\text {scal }}-2 \operatorname{Ric}_{N N}\right)\langle\not\rangle^{-1}\left(\left(\partial_{x^{3}} \chi^{2}\right)\left(\mathrm{R}_{\text {scal }}-2 \mathrm{Ric}_{N N}\right)\right) .
\end{aligned}
$$


The first term on the right-hand side of (2.15) equals by (2.14),

$$
\begin{aligned}
& \int_{0}^{r} \int_{\mathbb{R}^{2}} \chi^{2}\left(\mathrm{R}_{\text {scal }}-2 \operatorname{Ric}_{N N}\right)\langle\not \partial\rangle^{-1}\left(\chi^{2} a N\left(\mathrm{R}_{\text {scal }}-2 \operatorname{Ric}_{N N}\right)\right) \\
= & 2 \int_{0}^{r} \int_{\mathbb{R}^{2}} \chi^{2}\left(\mathrm{R}_{\text {scal }}-2 \operatorname{Ric}_{N N}\right)\langle\not \partial\rangle^{-1}\left(\chi^{2} a \operatorname{dijv} R \cdot N\right) \\
& -2 \int_{0}^{r} \int_{\mathbb{R}^{2}} \chi^{2}\left(\mathrm{R}_{s c a l}-2 \operatorname{Ric}_{N N}\right)\langle\not \partial\rangle^{-1}\left(\chi^{2} a\left(\operatorname{tr} \Theta \operatorname{Ric}_{N N}+\Theta^{A B} \operatorname{Ric}_{A B}+\operatorname{Ric}_{A N}\left(\nabla_{N} N\right)^{A}\right)\right) .
\end{aligned}
$$

The first term on the right-hand side of (2.16) is estimated by using that by Lemma 2.10, the definition dikv $X=\not j \not_{i} X_{j}$ for vectorfields $X$ and standard product estimates, see Lemma 2.5, we have for $\varepsilon>0$ sufficiently small,

$$
\|\langle\not \partial\rangle^{-1}\left(\chi^{2} a \text { diłvRic. } N\right)\left\|_{L^{2}\left(\mathbb{R}^{3}\right)} \leq C_{r^{\prime}, r}\right\| \chi \operatorname{Ric} \|_{L^{2}\left(\mathbb{R}^{2}\right)},
$$

and therefore

$$
\begin{aligned}
& 2 \int_{0}^{r} \int_{\mathbb{R}^{2}} \chi^{2}\left(\mathrm{R}_{\text {scal }}-2 \operatorname{Ric}_{N N}\right)\langle\not \partial\rangle^{-1}\left(\chi^{2} a \operatorname{dij} v R . N\right) \\
\lesssim & 2 \int_{0}^{r}\left\|\chi^{2}\left(\mathrm{R}_{\text {scal }}-2 \operatorname{Ric}_{N N}\right)\right\|_{L^{2}\left(\mathbb{R}^{2}\right)}\|\chi \operatorname{Ric}\|_{L^{2}\left(\mathbb{R}^{2}\right)} d x^{3} \\
\leq & C_{r^{\prime}, r}\|\operatorname{Ric}\|_{L^{2}\left(B^{+}(x, r)\right)}^{2} .
\end{aligned}
$$

The other terms are also estimated by Lemmas 2.10 and 2.5. Indeed, the second term of (2.15) involves only tangential derivatives analogously as the first term in (2.16), and the second term of (2.16) and third term of (2.15) are lower order. Details are left to the reader. We conclude that for $\varepsilon>0$ sufficiently small,

$$
\left\|\mathrm{R}_{\text {scal }}-2 \operatorname{Ric}_{N N}\right\|_{H^{-1 / 2}\left(\underline{B}^{+}\left(x, r^{\prime}\right)\right)}^{2} \leq C_{r^{\prime}, r}\|\operatorname{Ric}\|_{L^{2}\left(B^{+}(x, r)\right)}^{2} .
$$

This finishes the proof of Proposition 2.19, and hence the proof of (2.13).

\section{THE PRECISE VERSION OF THE MAIN RESUlT}

The following is our main result.

Theorem 3.1 (Existence of regular coordinates, version 2). Let $(M, g)$ be a compact, complete Riemannian 3-manifold with boundary such that

$$
\|\operatorname{Ric}\|_{L^{2}(M)}<\infty, r_{v o l}(M, 1)>0,\|\Theta\|_{L^{4}(\partial M)}<\infty .
$$


Then the following holds.

(1) $L^{2}$ regularity. There is $\varepsilon_{0}>0$ such that for every $0<\varepsilon<\varepsilon_{0}$, there is a radius

$$
r=r\left(\|\operatorname{Ric}\|_{L^{2}(M)},\|\Theta\|_{L^{4}(\partial M)}, r_{v o l}(M, 1), \varepsilon\right)>0
$$

such that for every point $p \in M$, there is a boundary harmonic chart ${ }^{5}$

$$
\varphi: B^{+}(x, r) \rightarrow U \subset M
$$

with $\varphi(x)=p$ in which, on $B^{+}(x, r)$,

$$
(1-\varepsilon) e_{i j} \leq g_{i j} \leq(1+\varepsilon) e_{i j}
$$

where $e_{i j}$ denotes the Euclidean metric. Moreover,

$$
r^{-1 / 2}\|\partial g\|_{\mathcal{L}^{2}\left(B^{+}(x, r)\right)}+r^{1 / 2}\left\|\partial^{2} g\right\|_{\mathcal{L}^{2}\left(B^{+}(x, r)\right)}<\varepsilon .
$$

(2) Higher regularity. Let $m \geq 1$ be an integer. Assuming higher regularity of Ric, we further have the higher regularity estimate

$$
\|g\|_{\mathcal{H}^{m+2}\left(B^{+}(x, r)\right)} \leq C_{r} \sum_{i=0}^{m}\left\|\nabla^{(i)} \operatorname{Ric}\right\|_{L^{2}(M)}+C_{m, r} \varepsilon .
$$

Remark 3.2. Cheeger-Gromov convergence theory for manifolds with boundary has been studied before, for example in [13], [4], [5] and [16], see Section 1.1. These results assume pointwise bounds on Ric, while in Theorem 3.1 we only assume $L^{2}$-bounds on Ric.

The proof of Theorem 5.1 is split into two. We prove part (1) in Theorem 5.1, and part (2) in Proposition 6.1.

\section{Convergence of Riemannian manifolds With Boundary}

In this section, we set up the Cheeger-Gromov theory on manifolds with boundary in low regularity. In the case of manifolds without boundary, these results are available for example in [20] and [21].

4.1. Convergence of functions, tensors and Riemannian manifolds. In this section, we introduce basic definitions of convergence.

Definition $4.1\left(C^{m, \alpha_{-}}\right.$and $H^{2}$-convergence of functions and tensors). Let $m \geq 0$ be an integer and $\alpha \in(0,1)$ a real. Let $(M, g)$ be a Riemannian manifold with boundary. Let $A \subset M$ be a pre-compact subset and $\left(U_{n}, \varphi_{n}\right)$ a finite number of fixed charts covering $A$. $A$ sequence of functions $\left(f_{i}\right)_{i \in \mathbb{N}}$ on $A$ is said to converge in $C^{m, \alpha}$ (in $H^{2}$ ) as $i \rightarrow \infty$, if for each $n$, the pullbacks $\left(\varphi_{n}\right)^{*} f_{i}$ converge in $C^{m, \alpha}$ (in $H^{2}$ ) as $i \rightarrow \infty$. The convergence of a sequence of tensors on $A$ in $C^{m, \alpha}$ (in $H^{2}$ ) is defined similarly.

The so-called pointed convergence of manifolds with boundary in $C^{m, \alpha}$ and $H^{2}$ is defined as follows.

\footnotetext{
${ }^{5}$ That is, the coordinates $x^{i}:=\left(\varphi^{-1}\right)^{i}: U \rightarrow \mathbb{R}$ are boundary harmonic.
} 
Definition 4.2 (Pointed $C^{m, \alpha_{-}}$and $H^{2}$-convergence of manifolds with boundary). $A$ sequence $\left(M_{i}, g_{i}, p_{i}\right)$ of pointed Riemannian manifolds with boundary is said to converge to a pointed Riemannian manifold with boundary $(M, g, p)$ in the pointed $C^{m, \alpha}$-topology (in the pointed $H^{2}$-topology) as $i \rightarrow \infty$, if for all $R>0$ there exists a bounded set $\Omega \subset M$ with smooth boundary containing the geodesic ball $B_{g}(p, R) \subset \Omega$ and embeddings $F_{i}: \Omega \rightarrow M_{i}$ for large $i$ such that $F_{i}(p)=p_{i}, B_{g}\left(p_{i}, R\right) \subset F_{i}(\Omega)$ and $\left(F_{i}\right)^{*} g_{i} \rightarrow g$ in the $C^{m, \alpha}$-topology (in the $H^{2}$-topology) on $\Omega$ as $i \rightarrow \infty$.

Remark 4.3. In this paper we consider sequences $\left(M_{i}, g_{i}\right)$ of smooth Riemannian manifolds with boundary that have uniform quantitative bounds, see for example the assumptions of Theorem 5.1. These sequences are then shown to convergence to a limit $(M, g)$ in a topology suited to the uniform bounds. The a priori regularity of the limit manifold is determined by the strength of the topology in which the convergence takes place.

4.2. The $C^{m, \alpha}$-norm of a Riemannian manifold. In this section, we define the $C^{m, \alpha_{-}}$ norm of a Riemannian manifold with boundary and state a compactness result for this norm.

Definition 4.4. Let $m \geq 0$ be an integer and $\alpha \in(0,1)$ a real. Let $(M, g, p)$ be a pointed smooth Riemannian 3-manifold with boundary. For two given reals $r>0$ and $Q \geq 0$, we write

$$
\|(M, g, p)\|_{C^{m, \alpha}, r} \leq Q
$$

if there exist a point $x \in \mathbb{H}^{+}$and a $C^{m+1, \alpha}$-regular chart $\varphi: B^{+}(x, r) \rightarrow U \subset M$ with $\varphi(x)=p$ such that

(n1) $|D \varphi| \leq e^{Q}$ on $B^{+}(x, r)$ and $\left|D \varphi^{-1}\right| \leq e^{Q}$ on $U{ }^{6}$

(n2) For all multi-indices $I$ with $0 \leq|I| \leq m$,

$$
\max _{i, j=1,2,3} r^{|I|+\alpha}\left[\partial^{I} g_{i j}\right]_{C^{0, \alpha}\left(B^{+}(x, r)\right)} \leq Q
$$

where $g_{i j}$ denotes the coordinate components of $g$ in the chart $\varphi$.

We set

$$
\|(M, g, p)\|_{C^{m, \alpha}, r}:=\inf \left\{Q \geq 0:\|(M, g, p)\|_{C^{m, \alpha}, r} \leq Q\right\},
$$

and let $\|(M, g, p)\|_{C^{m, \alpha}, r}=+\infty$ if the infimum does not exist. Define further

$$
\|(M, g)\|_{C^{m, \alpha}, r}:=\sup _{p \in M}\|(M, g, p)\|_{C^{m, \alpha}, r} .
$$

Remark 4.5. Let $(M, g, p)$ be a compact pointed smooth Riemannian manifold with boundary. Then, the $C^{m, \alpha}$-norm is finite for all $r>0$, that is,

$$
\|(M, g, p)\|_{C^{m, \alpha}, r}<\infty .
$$

${ }^{6}$ Here we denote

$$
|D \varphi|:=\sup _{\xi \in T \mathbb{R}^{3},|\xi|=1} \sqrt{g(D \varphi(\xi), D \varphi(\xi))},\left|D \varphi^{-1}\right|:=\sup _{X \in T M,|X|_{g}=1}\left|D \varphi^{-1}(X)\right| .
$$


Remark 4.6. The above condition (n1) is equivalent to

$$
e^{-2 Q} e_{i j} \leq g_{i j} \leq e^{2 Q} e_{i j},
$$

where e denotes the Euclidean metric.

We have the following properties of the $C^{m, \alpha}$-norm.

Proposition 4.7. Let $m \geq 0$ be an integer, $\alpha \in(0,1)$ a real and let $(M, g, p)$ be a pointed smooth Riemannian 3-manifold with boundary. Then the following holds.

(1) $\|(M, g, p)\|_{C^{m, \alpha}, r}=\left\|\left(M, \lambda^{2} g, p\right)\right\|_{C^{m, \alpha}, \lambda r}$ for all $\lambda>0$.

(2) The function $r \mapsto\|(M, g, p)\|_{C^{m, \alpha}, r}$ is increasing, continuous and converges to 0 as $r \rightarrow 0$.

(3) If $\|(M, g, p)\|_{C^{m, \alpha}, r}<Q$, then there exists a chart $(\varphi, U)$ such that the geodesic ball $B_{g}\left(p, e^{-Q} r\right) \subset U$.

Proof. We omit the proofs of the parts (1) and (2), because they are analogous to the proof of Proposition 4.12 below. Point (3) follows by using that with (4.1), the geodesic length in $M$ and the coordinate length in a chart can be compared.

For more details, see Proposition 11.3.1 in [21] for the case of manifolds without boundary.

We have the following compactness result.

Theorem 4.8 (Fundamental theorem of manifold convergence theory). For an integer $m \geq 0$ and reals $Q>0, \alpha \in(0,1)$ and $r>0$, let $\mathcal{M}^{m, \alpha}(Q, r)$ denote the class of pointed smooth Riemannian 3-manifolds with boundary $(M, g, p)$ such that

$$
\|(M, g)\|_{C^{m, \alpha}, r} \leq Q .
$$

The set $\mathcal{M}^{m, \alpha}(Q, r)$ is compact in the pointed $C^{m, \beta}$-topology for all $\beta<\alpha$.

As already noted in Section 3.1 in [5], the well-known proof for manifolds without boundary (see for example Theorem 11.3.6 in [21]) extends to manifolds with boundary without significant changes. We refer the reader to these literature results for a proof of Theorem 4.8 .

4.3. The boundary harmonic $H^{2}$-norm of a Riemannian manifold. In this section, we define the $H^{2}$-norm of a Riemannian manifold with boundary and state a precompactness result for this norm.

Definition 4.9. Let $(M, g, p)$ be a pointed smooth Riemannian 3-manifold with boundary. For two reals $r>0$ and $Q \geq 0$, we write

$$
\|(M, g, p)\|_{H^{2}, r}^{b . h .} \leq Q,
$$

if there exist a point $x \in \mathbb{H}^{+}$and a $H^{3}$-regular chart $\varphi: B^{+}(x, r) \rightarrow U \subset M$ with $\varphi(x)=p$ such that

(h1) $|D \varphi| \leq e^{Q}$ on $B^{+}(x, r)$ and $\left|D \varphi^{-1}\right| \leq e^{Q}$ on $U$. 
(h2) For all multi-indices $I$ with $1 \leq|I| \leq 2$,

$$
\max _{i, j=1,2,3} r^{|I|-3 / 2}\left\|\partial^{I} g_{i j}\right\|_{L^{2}\left(B^{+}(x, r)\right)} \leq Q
$$

where $g_{i j}$ denotes the coordinate components of $g$ in the chart $\varphi$.

(h3) The coordinates $\varphi^{-1}: U \rightarrow B^{+}(x, r)$ are boundary harmonic.

We set

$$
\|(M, g, p)\|_{H^{2}, r}^{b . h .}:=\inf \left\{Q \geq 0:\|(M, g, p)\|_{H^{2}, r}^{b . h .} \leq Q\right\}
$$

and let $\|(M, g, p)\|_{H^{2}, r}^{b . h .}=+\infty$ if the infimum does not exist. Let further

$$
\|(M, g)\|_{H^{2}, r}^{b . h}:=\sup _{p \in M}\|(M, g, p)\|_{H^{2}, r}^{b . h .} .
$$

Remark 4.10. Let $(M, g, p)$ be a compact pointed smooth Riemannian manifold with boundary $(M, g, p)$. Then the $H^{2}$-norm is finite for all $r>0$, that is,

$$
\|(M, g, p)\|_{H^{2}, r}^{b . h .}<\infty .
$$

Remark 4.11. The above condition (h1) is equivalent to

$$
e^{-2 Q} e_{i j} \leq g_{i j} \leq e^{2 Q} e_{i j}
$$

where e denotes the Euclidean metric.

We have the following properties of the boundary harmonic $H^{2}$-norm.

Proposition 4.12. Let $r>0$ be a real and let $(M, g, p)$ be a smooth pointed Riemannian 3-manifold with boundary and $p \in M$ a point. The following holds.

(1) $\left\|\left(M, \lambda^{2} g, p\right)\right\|_{H^{2}, \lambda r}^{b . h .}=\|(M, g, p)\|_{H^{2}, r}^{b . h .}$ for all reals $\lambda>0$.

(2) The function $r \mapsto\|(M, g, p)\|_{H^{2}, r}^{b . h}$ is increasing, continuous and converges to 0 as $r \rightarrow 0$.

(3) Let $\left(M_{i}, g_{i}, p_{i}\right)$ be a sequence of pointed smooth Riemannian manifolds with boundary and $(M, g, p)$ be a pointed smooth Riemannian manifold. If

$$
\left(M_{i}, g_{i}, p_{i}\right) \rightarrow(M, g, p)
$$

as $i \rightarrow \infty$ in the pointed $H^{2}$-topology, then it holds that

$$
\left\|\left(M_{i}, g_{i}, p_{i}\right)\right\|_{H^{2}, r}^{b . h .} \rightarrow\|(M, g, p)\|_{H^{2}, r}^{b . h .} .
$$

Proof. (1). For a real $\lambda>0$, consider the scaled

$$
\varphi_{\lambda}(x):=\varphi\left(\lambda^{-1} x\right): B^{+}(\lambda x, \lambda r) \rightarrow U \subset M, g_{\lambda}:=\lambda^{2} g .
$$

This pair satisfies the conditions (h1) and (h2) on $B^{+}(\lambda x, \lambda r)$ in Definition 4.9 with the same $Q<\infty$ as $(\varphi, g)$ on $B^{+}(x, r)$. Indeed, this follows because on $B^{+}\left(\lambda^{-1} x, \lambda^{-1} r\right)$

$$
\left(\varphi_{\lambda}\right)^{*} g_{\lambda}(x)=\varphi^{*} g(\lambda x)
$$

It remains to show that if $\varphi^{-1}$ is boundary harmonic for $g$, then $\left(\varphi_{\lambda}\right)^{-1}$ is boundary harmonic for $g_{\lambda}$. This follows by the invariance of the Laplace-Beltrami operator and is 
left to the reader. This finishes the proof of point (1).

(2). The monotonicity of the norm in $r>0$ follows directly by restriction. The continuity follows from the above scaling invariance by estimating for $\tilde{r}>0$

$$
\|(M, g)\|_{H^{2}, \tilde{r}}^{b . h .}=\left\|\left(M,\left(\frac{r}{\tilde{r}}\right)^{-2} g\right)\right\|_{H^{2}, r}^{b . h .} \leq \max \left\{Q+\left|\log \left(\frac{\tilde{r}}{r}\right)\right|,\left(\frac{\tilde{r}}{r}\right)^{2} Q\right\} .
$$

The estimate (4.2) implies that the norm is continuous in $r$, for more details see Propositions 11.3.2 and 11.3.5 in [21] for the case of manifolds without boundary.

It remains to show that as $r \rightarrow 0$,

$$
\|(M, g, p)\|_{H^{2}, r}^{b . h .} \rightarrow 0 .
$$

However, by Proposition 4.7 we have that as $r \rightarrow 0$,

$$
\|(M, g, p)\|_{C^{2,1 / 2}, r} \rightarrow 0 .
$$

Approximate these coordinates by boundary harmonic coordinates with appropriate prescribed Dirichlet and Neumann boundary data on the boundary, see Lemmas 2.15 and 2.17. The standard elliptic estimates in Corollaries A.4 and B.4 in the appendix show that these boundary harmonic coordinates admit the right bounds of size $\varepsilon$. Details are left to the reader.

(3). It suffices to prove the following two inequalities,

$$
\begin{gathered}
\limsup _{i \rightarrow \infty}\left\|\left(M_{i}, g_{i}, p_{i}\right)\right\|_{H^{2}, r}^{b . h .} \leq\|(M, g, p)\|_{H^{2}, r}^{b . h .}, \\
\liminf _{i \rightarrow \infty}\left\|\left(M_{i}, g_{i}, p_{i}\right)\right\|_{H^{2}, r}^{b . h .} \geq\|(M, g, p)\|_{H^{2}, r}^{b . h .} .
\end{gathered}
$$

In the following, we discuss (4.3), the estimate (4.4) is proved by an analogous argument left to the reader.

We recall from Definition 4.2 that the assumption

$$
\left(M_{i}, g_{i}, p_{i}\right) \rightarrow(M, g, p) \text { as } i \rightarrow \infty \text { in the pointed } H^{2} \text {-topology, }
$$

means that for each integer $i \geq 1$ and real $R>0$ a bounded open set $\Omega \subset M$ with smooth boundary such that $B_{g}(p, R) \subset \Omega$ together with an embedding $F_{i}: \Omega \rightarrow M_{i}$ such that $F_{i}(p)=p_{i}, B_{g_{i}}\left(p_{i}, R\right) \subset F_{i}(\Omega)$ and

$$
\left(F_{i}\right)^{*} g_{i} \rightarrow g \text { as } i \rightarrow \infty \text { in the } H^{2} \text {-topology on } \Omega \text {. }
$$

Using these $F_{i}$, locally define coordinates on $\left(M_{i}, g_{i}\right)$. Then approximate these coordinates on $\left(M_{i}, g_{i}\right)$ by boundary harmonic coordinates with appropriate Dirichlet and Neumann boundary conditions on the boundary, see Lemmas 2.15 and 2.17. By the standard elliptic estimates in Corollaries A.4 and B.4 in the appendix, and by the fact that due to (4.5) we have, schematically,

$$
\triangle_{\left(F_{i}\right)^{*} g_{i}} \rightarrow \triangle_{g} \text { as } i \rightarrow \infty
$$


it follows that these boundary harmonic coordinates on $\left(M_{i}, g_{i}\right)$ admit the right bounds. For more details, we refer to [20] where the case of manifolds without boundary is treated.

By Lemma 2.4, we have the following result. The proof is left to the reader.

Lemma 4.13. Let $r>0$. Let $(M, g)$ be a Riemannian 3-manifold with boundary. Then, for $\tilde{r}<r,\|(M, g)\|_{C^{0, \alpha}, \tilde{r}}$ for $\alpha \in(0,1 / 2)$ is bounded in terms of $\|(M, g)\|_{H^{2}, r}^{b . h}$.

As corollary of Theorem 4.8 and Lemma 4.13, we have the following pre-compactness result in the $H^{2}$-topology. The proof is left to the reader.

Corollary 4.14. Let $Q>0$ and $r>0$ be two reals. Let $\mathcal{H}^{2}(Q, r)$ denote the class of pointed Riemannian 3-manifolds $(M, g, p)$ with boundary such that $\|(M, g)\|_{H^{2}, r}^{b . h .} \leq Q$. Then $\mathcal{H}^{2}(Q, r)$ is pre-compact in the pointed $C^{0, \alpha}$-topology for $\alpha \in(0,1 / 2)$.

The next proposition shows that local $\mathrm{H}^{2}$-convergence in charts implies global pointed $H^{2}$-convergence of manifolds. The proof is based on the proof of Theorem 4.8 and left to the reader, see Lemma 4.5 in [20] for the case of manifolds without boundary.

Proposition 4.15. Let $r>0$ be a fixed real number. Let $(M, g, p)$ and $\left(M_{i}, g_{i}, p_{i}\right)$, for $i \geq 1$, be smooth Riemannian 3-manifolds with boundary. Suppose we have countable atlases of $M$ and $M_{i}$ for $i \geq 1$ consisting respectively of boundary harmonic $H^{3}$-charts

$$
\begin{gathered}
\varphi_{n}: B^{+}\left(x_{n}, r\right) \rightarrow U \subset M, \\
\varphi_{i n}: B^{+}\left(x_{n}, r\right) \rightarrow U_{i} \subset M_{i},
\end{gathered}
$$

such that

(1) $\varphi_{\text {in }} \rightarrow \varphi_{n}$ in the $H^{3}$-topology as $i \rightarrow \infty$,

(2) $\varphi_{n}$ and $\varphi_{\text {in }}$ satisfy (h1) and (h3) in Definition 4.9 for a uniform $Q>0$,

(3) $g_{\text {in }} \rightarrow g_{n}$ in $H^{2}\left(B^{+}\left(x_{n}, r\right)\right)$, where $g_{i n}:=\varphi_{\text {in }}{ }^{*} g_{i}$ and $g_{n}:=\varphi_{n}{ }^{*} g$.

Then, subsequentially,

$$
\left(M_{i}, g_{i}, p_{i}\right) \rightarrow(M, g, p) \text { as } i \rightarrow \infty \text { in the pointed } H^{2} \text {-topology. }
$$

\section{EXISTENCE OF BOUNDARY HARMONIC COORDINATES}

The following is the main result of this section.

Theorem 5.1 (Existence of boundary harmonic coordinates). Let $(M, h)$ be a compact smooth Riemannian 3-manifold with boundary satisfying

$$
\|\operatorname{Ric}\|_{L^{2}(M)} \leq \Lambda,\|\Theta\|_{L^{4}(\partial M)} \leq \Psi, r_{v o l}(M, 1) \geq v
$$

for constants $\Lambda \geq 0, \Psi \geq 0$ and $v>0$. Then, for every real $Q>0$, there exists an

$$
r=r(\Lambda, \Psi, v, Q)>0
$$

such that

$$
\|(M, h)\|_{H^{2}, r}^{b . h .} \leq Q
$$


Here $\Theta$ denotes the second fundamental form of $\partial M \subset M$, and $r_{\text {vol }}(M, 1)$ is the volume radius at scale 1 of $(M, h)$, see Definition 2.12.

Remark 5.2. Part (1) of Theorem 3.1 follows directly from Theorem 5.1 by picking $Q<\varepsilon$, see also point (3) of Proposition 4.7.

Theorem 5.1 is proved by contradiction and consists of four steps.

- Section 5.1. Existence of a converging subsequence in the pointed $C^{0, \alpha}$-topology for $\alpha \in(0,1 / 2)$.

- Section 5.2. Improvement to strong convergence in the $H^{2}$-topology.

- Section 5.3. Rigidity of the limit manifold.

- Section 5.4. The contradiction.

The Sections 5.2 and 5.3 are independent of each other and lead to the contradiction in Section 5.4.

5.1. Existence of a converging subsequence in the pointed $C^{0, \alpha}$-topology for $\alpha \in(0,1 / 2)$. In this section, we use the contradiction assumption to construct a sequence $\left(M_{i}, g_{i}, p_{i}\right)$ of smooth pointed Riemannian manifolds which converge in the pointed $C^{0, \alpha_{-}}$ topology for $\alpha \in(0,1 / 2)$ to a limit manifold $(M, g, p)$. Subsequently, in Section the strength of convergence is improved to $H^{2}$ and in Section 5.3 the limit manifold $(M, g, p)$ is analysed.

Assume for contradiction there exists

- a sequence of reals $r_{i}>0$ such that $\lim _{i \rightarrow \infty} r_{i}=0$,

- a sequence $\left(M_{i}, h_{i}\right)$ of compact smooth Riemannian manifolds with boundary which satisfy (5.1) and are such that for all $i$,

$$
\left\|\left(M_{i}, h_{i}\right)\right\|_{H^{2}, r_{i}}^{b . h .}>Q .
$$

The $\left(M_{i}, h_{i}\right)$ are smooth and compact, so their norm is finite. Therefore, using the continuity of the norm, see (2) of Proposition 4.12, we can decrease $r_{i}>0$ such that for all $i \geq 1$

$$
\left\|\left(M_{i}, h_{i}\right)\right\|_{H^{2}, r_{i}}^{b . h .}=Q .
$$

By (1) of Proposition 4.12, the conformally rescaled metric $g_{i}:=r_{i}^{-2} h_{i}$ satisfies

$$
\left\|\left(M_{i}, g_{i}\right)\right\|_{H^{2}, 1}^{b . h .}=\left\|\left(M_{i}, h_{i}\right)\right\|_{H^{2}, r_{i}}^{b . h .}=Q .
$$

First, by Definition 4.9 , this implies that for each $i \geq 1$ there is a point $p_{i} \in M_{i}$ such that

$$
\left\|\left(M_{i}, g_{i}, p_{i}\right)\right\|_{H^{2}, 1}^{b . h .} \geq Q / 2 \text {. }
$$

Second, by continuity of the norm, see Proposition 4.12 and (4.2), there is a $Q \leq \tilde{Q}<\infty$ such that for all $i \geq 1$

$$
\left\|\left(M_{i}, g_{i}\right)\right\|_{H^{2}, 2}^{b . h .}=\tilde{Q}>Q
$$


At this point, we continue at scale $r=2$ because this gives us the leeway to apply interior elliptic estimates in $\partial M$ to deduce estimates at scale $r=1$.

By (5.2) and Corollary 4.14, there is a pointed Riemannian manifold with boundary $(M, g, p)$ such that subsequentially as $i \rightarrow \infty$,

$$
\left(M_{i}, g_{i}, p_{i}\right) \rightarrow(M, g, p) \text { as } i \rightarrow \infty \text { in the } C^{0, \alpha} \text {-topology }
$$

for any $\alpha \in(0,1 / 2)$. This convergence comes together with countable atlases of $M$ and $M_{i}, i \geq 1$, consisting of boundary harmonic $H^{3}$-charts

$$
\begin{aligned}
\varphi_{n} & : B^{+}\left(x_{n}, 2\right) \rightarrow U \subset M, \\
\varphi_{\text {in }}: B^{+}\left(x_{n}, 2\right) & \rightarrow U_{i n} \subset M_{i},
\end{aligned}
$$

respectively, where each $\varphi_{\text {in }}$ satisfies (h1)-(h3) of Definition 4.9 with constant $\tilde{Q}$, see (5.2), and for $\alpha \in(0,1 / 2)$,

$$
\varphi_{i n} \rightarrow \varphi_{n} \text { as } i \rightarrow \infty \text { in the } C^{1, \alpha} \text {-topology. }
$$

Denote the coordinate components of $g_{i}, i \geq 1$, and $g$ on $B^{+}\left(x_{n}, 2\right)$ by

$$
g_{\text {in }}:=\left(\varphi_{i n}\right)^{*} g_{i}, g_{n}:=\left(\varphi_{n}\right)^{*} g \text {. }
$$

We now show three important properties of the constructed sequence.

(1) By the relation $g_{i}:=r_{i}^{-2} h_{i}$ and the uniform bounds (5.1), it follows that as $i \rightarrow \infty$,

$$
\begin{aligned}
\left\|\operatorname{Ric}_{i n}\right\|_{\mathcal{L}^{2}\left(B^{+}\left(x_{n}, 2\right)\right)} & \rightarrow 0 \\
\left\|\Theta_{i n}\right\|_{\mathcal{L}^{4}\left(\underline{B}^{+}\left(x_{n}, 2\right)\right)} & \rightarrow 0
\end{aligned}
$$

where $\Theta_{i n}$ denotes the second fundamental forms of $\underline{B}^{+}\left(x_{n}, 2\right)$ with respect to $g_{i}$. In particular, it follows that for the limit Riemannian manifold $(M, g)$,

$$
\text { Ric }=0 \text { on } M \text { and } \Theta=0, K=0 \text { on } \partial M,
$$

where $K$ denotes the Gauss curvature of $\partial M$.

(2) By (5.2), the compactness of bounded closed sets for the weak topology and Lemma 2.4 , it holds that for each $n$, (subsequentially) as $i \rightarrow \infty$,

$$
\begin{array}{ll}
g_{\text {in }} \rightarrow g_{n} & \text { in } \mathcal{H}^{2}\left(B^{+}\left(x_{n}, 2\right)\right), \\
g_{\text {in }} \rightarrow g_{n} & \text { in } \mathcal{H}^{1}\left(B^{+}\left(x_{n}, 2\right)\right), \\
g_{\text {in }} \rightarrow g_{n} & \text { in } \mathcal{C}^{0}\left(B^{+}\left(x_{n}, 2\right)\right), \\
\not_{\text {in }} \rightarrow \not_{n} & \text { in } \mathcal{H}^{5 / 4}\left(\underline{B}^{+}\left(x_{n}, 2\right)\right)
\end{array}
$$

In particular, we have for the limit metric

$$
\left\|g_{n}\right\|_{\mathcal{H}^{2}\left(B^{+}\left(x_{n}, 2\right)\right)} \lesssim \tilde{Q} .
$$


(3) We have the following volume growth estimate on $(M, g)$. For all $r>0$, it holds that

$$
\operatorname{vol}_{g}\left(B_{g}(p, r)\right) \geq \frac{4 \pi}{3} v r^{3} .
$$

Indeed, since $\left(M_{i}, h_{i}\right)$ satisfies (5.1), for each integer $i \geq 1$ and point $p_{i} \in M_{i}$, we have

$$
\operatorname{vol}_{h_{i}}\left(B_{h_{i}}\left(p_{i}, r\right)\right) \geq \frac{4 \pi}{3} v r^{3} \text { for all } r \leq 1 .
$$

For the rescaled $\left(M_{i}, g_{i}\right)$ this implies that for all reals $0 \leq r \leq\left(r_{i}\right)^{-1}$ and points $p_{i} \in M_{i}$,

$$
\operatorname{vol}_{g_{i}}\left(B_{g_{i}}\left(p_{i}, r\right)\right)=\frac{1}{r_{i}^{3}} \operatorname{vol}_{h_{i}}\left(B_{h_{i}}\left(p_{i}, r r_{i}\right)\right) \geq \frac{1}{r_{i}^{3}} \frac{4 \pi}{3} v\left(r r_{i}\right)^{3}=\frac{4 \pi}{3} v r^{3}
$$

where we used that $r r_{i} \leq 1$ to apply (5.8).

Using that $\frac{1}{r_{i}} \rightarrow \infty$ as $i \rightarrow \infty$, and $\left(M_{i}, g_{i}, p_{i}\right) \rightarrow(M, g, p)$ as $i \rightarrow \infty$ in the pointed $C^{0, \alpha}$-topology, we have for each fixed $r \geq 0$ (for $i \geq 1$ sufficiently large) as $i \rightarrow \infty$,

$$
\operatorname{vol}_{g_{i}}\left(B_{g}\left(p_{i}, r\right)\right) \rightarrow \operatorname{vol}_{g}\left(B_{g}(p, r)\right),
$$

see also the proof of Lemma 11.4.9 in [21] for the case of manifolds without boundary. This proves (5.7).

5.2. Improvement to strong convergence in the pointed $H^{2}$-topology. In this section, we prove that

$$
\left(M_{i}, g_{i}, p_{i}\right) \rightarrow(M, g, p) \text { in the pointed } H^{2} \text {-topology as } i \rightarrow \infty .
$$

To prove (5.9), it suffices by Proposition 4.15 to show that locally, in the charts $\varphi_{\text {in }}$ and $\varphi_{n}$, see $(5.3)$,

$$
g_{\text {in }} \rightarrow g_{n} \text { in } \mathcal{H}^{2}\left(B^{+}\left(x_{n}, 1\right)\right) \text { as } i \rightarrow \infty .
$$

Indeed, Proposition 4.15 can be applied because the charts $\varphi_{i n}$ have uniform bounds, see (5.2), and it is shown independently in Section 5.3 that $(M, g, p)$ is a smooth pointed Riemannian 3-manifold.

For the rest of this section, we prove (5.10) for a fixed $n$ and hence leave away the index $n$ for ease of presentation.

We prove (5.10) in three steps.

- Section 5.2.1. Strong convergence of the Gauss curvature of $\partial M$.

- Section 5.2.2. Strong convergence of the coordinate components $\not_{A B}$ and $g_{A B}$. Here $\not g$ denotes the induced metric on $\partial M$, see also (2.5).

- Section 5.2.3. Strong convergence of the components $g^{33}$ and $g^{A 3}$. 
In the following calculations in Sections 5.2.1-5.2.3, we tacitly use that by (5.2),

$$
\left(g_{i}\right)^{l m},\left(g_{i}\right)_{l m}, \sqrt{\operatorname{det} g_{i}} \text { for } l, m=1,2,3, i \geq 1
$$

are uniformly bounded in $B^{+}(x, 2)$.

5.2.1. Strong convergence of the Gauss curvature. Let $K$ and $K_{i}$ be the Gauss curvature of $\partial M \subset(M, g)$ and $\partial M_{i} \subset\left(M_{i}, g_{i}\right)$, respectively. In this section we show that

$$
K_{i} \rightarrow K \text { in } H^{-1 / 2}\left(\underline{B}^{+}(x, 11 / 6)\right) \text { as } i \rightarrow \infty .
$$

By (5.5), $K=0$ on $\partial M$, and therefore it suffices to prove that

$$
\left\|K_{i}\right\|_{H^{-1 / 2}\left(\underline{B}^{+}(x, 11 / 6)\right)} \rightarrow 0 \text { as } i \rightarrow \infty .
$$

However, (5.11) follows from (5.4) with the trace estimate (2.13), that is,

$$
\left\|K_{i}\right\|_{H^{-1 / 2}\left(\underline{B}^{+}(x, 11 / 6)\right)} \lesssim\left\|\operatorname{Ric}_{i}\right\|_{L^{2}\left(B^{+}(x, 2)\right)}+\left\|\Theta_{i}\right\|_{L^{4}\left(\underline{B}^{+}(x, 2)\right)} .
$$

5.2.2. Strong convergence of $\oiint_{A B}$ and $g_{A B}$ components. In this section we show that as $i \rightarrow \infty$,

$$
\begin{aligned}
\left\|g_{i A B}-\not g_{A B}\right\|_{H^{3 / 2}\left(\underline{B}^{+}(x, 10 / 6)\right)} & \rightarrow 0, \\
\left\|g_{i A B}-g_{A B}\right\|_{H^{2}\left(B^{+}(x, 9 / 6)\right)} & \rightarrow 0 .
\end{aligned}
$$

First consider (5.12). By Lemma 2.15 and (5.5), $\not \phi_{i}$ and $\not g$ satisfy on $\underline{B}^{+}(x, 2)$

$$
\begin{aligned}
\frac{1}{2} \phi_{i} \phi_{i A B}+\phi_{i A B} & =-K_{i}, \\
\frac{1}{2} \not \oiint_{A B}+\phi_{A B} & =0 .
\end{aligned}
$$

By the interior elliptic estimate of Theorem B.1 applied to (5.14),

$$
\begin{aligned}
& \left\|\phi_{i A B}-\not \phi_{A B}\right\|_{H^{3 / 2}\left(\underline{B}^{+}(x, 10 / 6)\right)} \\
& \lesssim\left\|\not_{i}\left(\phi_{i A B}-\not \phi_{A B}\right)\right\|_{H^{-1 / 2}\left(\underline{B}^{+}(x, 11 / 6)\right)}+\left\|\phi_{i A B}-\not \phi_{A B}\right\|_{H^{1 / 2}\left(\underline{B}^{+}(x, 11 / 6)\right)} \\
& \lesssim\left\|\left(\bigotimes_{i}-\not\right) \not_{A B}\right\|_{H^{-1 / 2}\left(\underline{B}^{+}(x, 11 / 6)\right)}+\left\|\phi_{i A B}-\phi_{A B}\right\|_{H^{-1 / 2}\left(\underline{B}^{+}(x, 11 / 6)\right)} \\
& +\left\|K_{i}\right\|_{H^{-1 / 2}\left(\underline{B}^{+}(x, 11 / 6)\right)}+\left\|\phi_{i A B}-\oiint_{A B}\right\|_{H^{1 / 2}\left(\underline{B}^{+}(x, 11 / 6)\right)} \text {. }
\end{aligned}
$$

By (5.6), (5.11) and Lemmas 2.4 and 2.16, as $i \rightarrow \infty$,

$$
\begin{aligned}
\left\|\left(\phi_{i}-\not \Delta\right) \phi_{A B}\right\|_{H^{-1 / 2}\left(\underline{B}^{+}(x, 11 / 6)\right)} & \rightarrow 0, \\
\left\|\phi_{i A B}-\phi_{A B}\right\|_{H^{-1 / 2}\left(\underline{B}^{+}(x, 11 / 6)\right)} & \rightarrow 0, \\
\left\|K_{i}\right\|_{H^{-1 / 2}\left(\underline{B}^{+}(x, 11 / 6)\right)} & \rightarrow 0 .
\end{aligned}
$$

Together (5.15) and (5.16) prove that

$$
\left\|\not y_{i A B}-\not \phi_{A B}\right\|_{H^{3 / 2}\left(\underline{B}^{+}(x, 10 / 6)\right)} \rightarrow 0 \text { as } i \rightarrow \infty,
$$

which finishes the proof of (5.12). 
We turn to the proof of (5.13). By Lemma 2.15 and $(5.5)$, it holds that on $B^{+}(x, 2)$,

$$
\begin{aligned}
\frac{1}{2} \triangle_{i} g_{i A B}+Q_{i A B} & =-\operatorname{Ric}_{i A B}, \\
\frac{1}{2} \triangle g_{A B}+Q_{A B} & =0 .
\end{aligned}
$$

By the global elliptic estimates of Theorem A.1 with (5.18), we have

$$
\begin{aligned}
& \left\|g_{i A B}-g_{A B}\right\|_{H^{2}\left(B^{+}(x, 9 / 6)\right)} \\
\lesssim & \left\|\triangle_{i}\left(g_{i A B}-g_{A B}\right)\right\|_{L^{2}\left(B^{+}(x, 10 / 6)\right)}+\left\|g_{i A B}-g_{A B}\right\|_{H^{3 / 2}\left(\underline{B}^{+}(x, 10 / 6)\right)} \\
\lesssim & \left\|\left(\triangle_{i}-\triangle\right) g_{A B}\right\|_{L^{2}\left(B^{+}(x, 10 / 6)\right)}+\left\|Q_{i A B}-Q_{A B}\right\|_{L^{2}\left(B^{+}(x, 10 / 6)\right)} \\
& +\left\|\operatorname{Ric}_{i}\right\|_{L^{2}\left(B^{+}(x, 10 / 6)\right)}+\left\|g_{i A B}-\not g_{A B}\right\|_{H^{3 / 2}\left(\underline{B}^{+}(x, 10 / 6)\right)} \\
& +\left\|g_{i A B}-g_{A B}\right\|_{H^{1}\left(B^{+}(x, 10 / 6)\right)},
\end{aligned}
$$

where we used that $\mathscr{g}_{i A B}=g_{i A B}$ on $\underline{B}^{+}(x, 2)$. By (5.17), (5.4), (5.6) and using that by Lemmas 2.4 and 2.16, as $i \rightarrow \infty$,

$$
\begin{aligned}
\left\|\left(\triangle_{i}-\triangle\right) g_{A B}\right\|_{L^{2}\left(B^{+}(x, 10 / 6)\right)} & \rightarrow 0, \\
\left\|Q_{i A B}-Q_{A B}\right\|_{L^{2}\left(B^{+}(x, 10 / 6)\right)} & \rightarrow 0, \\
\left\|\operatorname{Ric}_{i}\right\|_{L^{2}\left(B^{+}(x, 10 / 6)\right)} & \rightarrow 0,
\end{aligned}
$$

it follows that

$$
\left\|g_{i A B}-g_{A B}\right\|_{H^{2}\left(B^{+}(x, 9 / 6)\right)} \rightarrow 0 \text { as } i \rightarrow \infty,
$$

which finishes the proof of (5.13).

5.2.3. Strong convergence of $g^{33}$ and $g^{3 A}$ components. In this section, we show that as $i \rightarrow \infty$,

$$
\begin{aligned}
\left\|g_{i}^{33}-g^{33}\right\|_{H^{2}\left(B^{+}(x, 8 / 6)\right)} & \rightarrow 0, \\
\left\|g_{i}^{3 A}-g^{3 A}\right\|_{H^{2}\left(B^{+}(x, 7 / 6)\right)} & \rightarrow 0 .
\end{aligned}
$$

On the one hand, by Lemma 2.15 and (5.5), we have on $B^{+}(x, 2)$ for $j=1,2,3$

$$
\begin{aligned}
& \frac{1}{2} \triangle_{g_{i}} g_{i}^{3 j}+Q_{i}^{3 j}=-\operatorname{Ric}_{i}^{33}, \\
& \frac{1}{2} \triangle_{g} g^{3 j}+Q^{3 j}=0 .
\end{aligned}
$$

On the other hand, by Lemma 2.17 , on $\underline{B}^{+}(x, 2)$ for all $i \geq 1$,

$$
\begin{aligned}
N_{i}\left(g_{i}^{33}\right) & =2 \operatorname{tr} \Theta_{i} g_{i}^{33} \\
N_{i}\left(g_{i}^{3 A}\right) & =\operatorname{tr} \Theta_{i} g_{i}^{3 A}-\frac{1}{2} \frac{1}{\sqrt{g_{i}^{33}}} g_{i}^{A m} \partial_{m} g_{i}^{33} .
\end{aligned}
$$


First consider (5.19). By the elliptic estimates of Theorem A.6 with (5.21) we have

$$
\begin{aligned}
& \left\|g_{i}^{33}-g^{33}\right\|_{H^{2}\left(B^{+}(x, 8 / 6)\right)} \\
\lesssim & \left\|\triangle_{g}\left(g^{33}-g_{i}^{33}\right)\right\|_{L^{2}\left(B^{+}(x, 9 / 6)\right)}+\left\|g^{33}-g_{i}^{33}\right\|_{H^{1}\left(B^{+}(x, 9 / 6)\right)}+\left\|N\left(g^{33}-g_{i}^{33}\right)\right\|_{H^{1 / 2}\left(\underline{B}^{+}(x, 9 / 6)\right)} \\
\lesssim & \left\|\left(\triangle_{g}-\triangle_{g_{i}}\right) g^{33}\right\|_{L^{2}\left(B^{+}(x, 9 / 6)\right)}+\left\|Q_{i}^{33}-Q^{33}\right\|_{L^{2}\left(B^{+}(x, 9 / 6)\right)}+\left\|\operatorname{Ric}_{i}^{33}\right\|_{L^{2}\left(B^{+}(x, 9 / 6)\right)} \\
& +\left\|g^{33}-g_{i}^{33}\right\|_{H^{1}\left(B^{+}(x, 9 / 6)\right)}+\left\|N\left(g^{33}-g_{i}^{33}\right)\right\|_{H^{1 / 2}\left(\underline{B}^{+}(x, 9 / 6)\right)}
\end{aligned}
$$

The last term on the right-hand side of (5.23) is bounded by (5.22) and Lemmas 2.4 and 2.5 as

$$
\begin{aligned}
& \left\|N\left(g^{33}-g_{i}^{33}\right)\right\|_{H^{1 / 2}\left(\underline{B}^{+}(x, 9 / 6)\right)} \\
\lesssim & \left\|g^{33} \operatorname{tr} \Theta-g_{i}^{33} \operatorname{tr} \Theta_{i}\right\|_{H^{1 / 2}\left(\underline{B}^{+}(x, 9 / 6)\right)}+\left\|\left(N-N_{i}\right) g^{33}\right\|_{H^{1 / 2}\left(\underline{B}^{+}(x, 9 / 6)\right)} \\
\lesssim & \left\|\operatorname{tr} \Theta_{i}\right\|_{H^{1 / 2}\left(\underline{B}^{+}(x, 9 / 6)\right)}+\left\|g^{3 j}-g_{i}^{3 j}\right\|_{H^{5 / 4}\left(\underline{B}^{+}(x, 9 / 6)\right)}\left\|\partial g^{33}\right\|_{H^{1 / 2}\left(\underline{B}^{+}(x, 9 / 6)\right)} \\
\lesssim & \left\|\operatorname{tr} \Theta_{i}\right\|_{H^{1 / 2}\left(\underline{B}^{+}(x, 9 / 6)\right)}+\left\|g^{3 j}-g_{i}^{3 j}\right\|_{H^{5 / 4}\left(\underline{B}^{+}(x, 9 / 6)\right)}\left\|g^{33}\right\|_{H^{2}\left(B^{+}(x, 9 / 6)\right)},
\end{aligned}
$$

where we used that $\operatorname{tr} \Theta=0$ and $N=-\left(g^{33}\right)^{-1 / 2} \nabla x^{3}$, see (2.6) and (5.5).

By (5.6) and Lemmas 2.4, 2.5 and 2.16, as $i \rightarrow \infty$,

$$
\begin{aligned}
\left\|\left(\triangle_{g}-\triangle_{g_{i}}\right) g^{33}\right\|_{L^{2}\left(B^{+}(x, 9 / 6)\right)} & \rightarrow 0, \\
\left\|Q_{i}^{33}-Q^{33}\right\|_{L^{2}\left(B^{+}(x, 9 / 6)\right)} & \rightarrow 0 .
\end{aligned}
$$

Moreover, using that in general, $\left.\Theta_{A B}=\frac{1}{2 a} \partial_{3}\left(g_{A B}\right)-\frac{1}{2 a}\left(\mathcal{A}_{\beta} \not\right)\right)_{A B}$, it follows from (5.13) that

$$
\left\|\operatorname{tr} \Theta_{i}-\operatorname{tr} \Theta\right\|_{H^{1 / 2}(x, 9 / 6)} \rightarrow 0 \text { as } i \rightarrow \infty .
$$

Therefore, plugging (5.24) into (5.23) and using (5.25) and (5.26), we have

$$
\left\|g^{33}-g_{i}^{33}\right\|_{H^{2}\left(B^{+}(x, 8 / 6)\right)} \rightarrow 0 \text { as } i \rightarrow \infty
$$

which finishes the proof of (5.19).

The proof of (5.20) is analogous and left to the reader.

To summarise, in the above we proved that, as $i \rightarrow \infty$,

$$
\left\|g_{i A B}-g_{A B}\right\|_{H^{2}\left(B^{+}(x, 9 / 6)\right)}+\left\|g_{i}^{3 A}-g^{3 A}\right\|_{H^{2}\left(B^{+}(x, 8 / 6)\right)}+\left\|g_{i}^{3 A}-g^{3 A}\right\|_{H^{2}\left(B^{+}(x, 7 / 6)\right)} \rightarrow 0 .
$$

By Lemma 2.18, this implies

$$
\left\|g_{i}-g\right\|_{\mathcal{H}^{2}\left(B^{+}(x, 1)\right)} \rightarrow 0 \text { as } i \rightarrow \infty
$$

which finishes the proof of (5.10). 
5.3. Rigidity of the limit manifold. In this section we prove that the limit manifold

$$
(M, g) \simeq\left(\mathbb{H}^{+}, e\right) .
$$

The proof of (5.27) has three steps.

- Section 5.3.1. Proof of smoothness of $g$.

- Section 5.3.2. Doubling of $(M, g)$ and construction of a smooth atlas.

- Section 5.3.3. Rigidity of the limit manifold.

5.3.1. Proof of smoothness of $g$. We recall from Section 5.1 that for all points $p \in M$ in the limit manifold $(M, g)$, there exists a boundary harmonic $H^{3}$-chart $\varphi: B^{+}(x, 1) \rightarrow U \subset M$ with $\varphi(x)=p$ such that for the constant $0<\tilde{Q}<\infty$,

- on $B^{+}(x, 1)$,

$$
e^{-2 \tilde{Q}} e_{i j} \leq g_{i j} \leq e^{2 \tilde{Q}} e_{i j}
$$

- for all multi-indices $I$ with $1 \leq|I| \leq 2$,

$$
\max _{i, j=1,2,3}\left\|\partial^{I} g_{i j}\right\|_{L^{2}\left(B^{+}(x, 1)\right)} \leq \tilde{Q} .
$$

By Lemma 2.15, the metric components $g_{i j}$ satisfy

$$
\begin{aligned}
\frac{1}{2} \triangle_{g} g_{i j}+Q_{i j} & =0 \text { on } B^{+}(x, 1), \\
\frac{1}{2} \triangle_{g} \phi_{A B}+\phi_{A B} & =0 \text { on } \underline{B}^{+}(x, 1) .
\end{aligned}
$$

These equations can be used to bootstrap regularity to $g \in C^{\infty}\left(B^{+}(x, 1)\right)$. We postpone this bootstrapping to Section 6, see Proposition 6.1. It follows in particular that $(M, g)$ is a smooth Ricci-flat (and hence flat) Riemannian 3-manifold with boundary.

Remark 5.3. The modulus of continuity of $g$ in $C^{k}$, for $k \geq 0$, is controlled in terms of $\tilde{Q}$, independently of the point $p \in M$.

5.3.2. The manifold double of $(M, g)$. In this section, we define the manifold double $M_{\text {double }}$ of $(M, g)$ and show that it is a smooth Riemannian manifold without boundary.

The manifold double $M_{\text {double }}$ is topologically defined as

$$
M_{\text {double }}:=\{0,1\} \times M
$$

where the boundaries $\{0\} \times \partial M$ and $\{1\} \times \partial M$ are identified.

Given that $(M, g)$ is a Riemannian manifold with boundary, the sets $\{0\} \times M$ and $\{1\} \times M$, equipped with metric $g$, are also Riemannian manifolds with boundary.

In the following, we construct interior charts of $M_{\text {double }}$ centered on points $p \in\{0\} \times$ $\partial M=\{1\} \times \partial M$ and show that the metric $g$ on $M$ extends smoothly to a Riemannian metric on $M_{\text {double }}$.

Let $p \in\{0\} \times \partial M$. By Section 5.3.1, there is a boundary harmonic chart

$$
\varphi: B^{+}(0,1) \rightarrow U \subset M
$$


with $\varphi(0)=p$ such that $g_{i j} \in C^{\infty}\left(B^{+}(0,1)\right)$.

Using this smoothness, we can construct so-called Gaussian normal coordinates $\left(z^{1}, z^{2}, z^{3}\right)$ in a neighbourhood $\mathcal{U} \subset\{0\} \times M$ of $p$, see for example Section 3.3 in [27]. They are such that for a small real $\delta>0$,

$$
\begin{aligned}
\mathcal{U} & =\left\{z \in \underline{B}^{+}(0,1 / 2) \times[0, \delta]\right\}, \\
\mathcal{U} \cap \partial M & =\left\{z \in \underline{B}^{+}(0,1 / 2) \times\{0\}\right\}, \\
\nabla_{\partial_{z^{3}}} \partial_{z^{3}} & =0, \\
\left.\partial_{z^{3}}\right|_{\mathcal{U} \cap \partial M} & \text { is normal to } \mathcal{U} \cap \partial M, \\
g\left(\partial_{z^{3}}, \partial_{z^{3}}\right) & =1 .
\end{aligned}
$$

We note that the scale $\delta>0$ can be bounded from below by the modulus of continuity of the metric $g$, that is, $\tilde{Q}$, see also Remark 5.3. In these coordinates, the metric $g$ is given by

$$
g=\left(d z^{3}\right)^{2}+\not g_{A B}^{z} d z^{A} d z^{B},
$$

where $\phi^{z}$ denotes the induced metric on level sets of $z^{3}$. These coordinates are also called zero-shift coordinates, because $\beta=0$ in $\underline{B}^{+}(0,1 / 2) \times[0, \delta]$, see $(2.5)$.

These coordinates are a local boundary chart of $\{0\} \times M$ around $p$. By applying the same construction around $p$ in $\{1\} \times M$ and identifying the coordinates $z^{1}, z^{2}$ on $\partial M$, we can extend the above coordinates to coordinates on $M_{\text {double, }}$, with chart

$$
\varphi: \underline{B}^{+}(0,1 / 2) \times[-\delta, \delta] \rightarrow M_{\text {double }} .
$$

Indeed, the geodesic construction of $z^{3}$ and the consequent transport of $\left(z^{1}, z^{2}\right)$ along $z^{3}$ yields smooth coordinates around $p$ in $M_{\text {double }}$.

The pullback of $g$ by the chart $\varphi: \underline{B}^{+}(0,1 / 2) \times[-\delta, \delta] \rightarrow M_{\text {double }}$ in (5.29) is given by

$$
(\varphi)^{*} g_{i j}\left(z^{1}, z^{2}, z^{3}\right):= \begin{cases}g_{i j}\left(z^{1}, z^{2}, z^{3}\right), & \text { if } z^{3} \geq 0 \\ g_{i j}\left(z^{1}, z^{2},-z^{3}\right) & \text { else }\end{cases}
$$

where $g_{i j}$ on $\underline{B}^{+}(0,1 / 2) \times[0, \delta]$ are the pullback components of $g$ as in (5.28).

Claim 5.4. The pullback metric $g$ in $(5.30)$ is smooth on $\underline{B}^{+}(0,1 / 2) \times[-\delta, \delta]$.

It suffices to prove that on $\underline{B}^{+}(0,1 / 2) \times\{0\}$

$$
\partial_{z^{3}}^{m} g_{A B}=0
$$

for all $m \geq 0$.

On the one hand, on all of $\underline{B}^{+}(0,1 / 2) \times[-\delta, \delta]$, it holds that

$$
g\left(\partial_{z^{A}}, \nabla_{\partial_{z^{3}}} \partial_{z^{B}}\right)=\frac{1}{2}\left(\partial_{z^{A}} g_{3 B}+\partial_{z^{3}} g_{A B}-\partial_{z^{B}} g_{3 A}\right)=\frac{1}{2} \partial_{z^{3}} g_{A B}
$$


where we used that $g_{3 A}=0$ in the zero-shift coordinates. Moreover, differentiating (5.32) by $\partial_{z^{3}}$ yields

$$
\begin{aligned}
\frac{1}{2} \partial_{z^{3}}^{2} g_{A B} & =g\left(\nabla_{\partial_{z^{3}}} \partial_{z^{A}}, \nabla_{\partial_{z^{3}}} \partial_{z^{B}}\right)+g\left(\partial_{z^{A}}, \nabla_{\partial_{z^{3}}}^{2} \partial_{z^{B}}\right) \\
& =g\left(\nabla_{\partial_{z^{3}}} \partial_{z^{A}}, \nabla_{\partial_{z^{3}}} \partial_{z^{B}}\right)+g\left(\partial_{z^{A}}, \nabla_{\partial_{z^{3}}} \nabla_{\partial_{z^{B}}} \partial_{z^{3}}\right) \\
& =g\left(\nabla_{\partial_{z^{3}}} \partial_{z^{A}}, \nabla_{\partial_{z^{3}}} \partial_{z^{B}}\right)+g\left(\partial_{z^{A}}, \operatorname{Rm}\left(\partial_{z^{3}}, \partial_{z^{B}}\right) \partial_{z^{3}}\right)+g\left(\partial_{z^{A}}, \nabla_{\partial_{z^{B}}} \nabla_{\partial_{z^{3}}} \partial_{z^{3}}\right) \\
& =g\left(\nabla_{\partial_{z^{3}}} \partial_{z^{A}}, \nabla_{\partial_{z^{3}}} \partial_{z^{B}}\right),
\end{aligned}
$$

where we used that $(M, g)$ is flat and $\partial_{z^{3}}$ geodesic.

On the other hand, because $\beta=0$ and $\Theta=0$ on $z^{3}=0$, it follows by (2.8) that on $\left\{z^{3}=0\right\}$

$$
\partial_{z^{3}} g_{A B}=0, \quad \nabla_{\partial_{z^{3}}} \partial_{z^{B}}=0 .
$$

This shows that $\partial_{z^{3}}^{2} g_{A B}=0$ at $\left\{z^{3}=0\right\}$. The statement (5.31) follows by continued differentiation of (5.32). This finishes the proof of Claim 5.4. This finishes the proof that $\left(M_{\text {double }}, g\right)$ is a smooth flat Riemannian 3-manifold without boundary. Further, by the fact that $(M, g)$ is a complete Riemannian manifold, it follows that $\left(M_{\text {double }}, g\right)$ is also complete.

5.3.3. Conclusion of the rigidity of the limit manifold. In this section we conclude that

$$
\left(M_{\text {double }}, g\right) \simeq\left(\mathbb{R}^{3}, e\right) .
$$

We recall that in the previous sections, we showed that $\left(M_{\text {double }}, g\right)$ is a complete smooth flat Riemannian 3-manifold without boundary. We further recall that by (5.7) there is a $v>0$ such that for all $r>0$ and $p \in M$,

$$
\operatorname{vol}_{g}\left(B_{g}(p, r)\right) \geq \frac{4 \pi}{3} v r^{3} .
$$

This lower bound on the volume growth continues to hold on the doubled $\left(M_{\text {double }}, g\right)$.

Proposition 5.5. Let $(M, g)$ be a complete smooth flat Riemannian 3-manifold such that there is a $v>0$ so that for all reals $r>0$ and points $p \in M$,

$$
\operatorname{vol}_{g}\left(B_{g}(p, r)\right) \geq v r^{3} \text {. }
$$

Then,

$$
(M, g) \simeq\left(\mathbb{R}^{3}, e\right)
$$

A proof of this proposition can be found at the end of the proof of Lemma 11.4.9 in [21], see also Theorem 5.4 in [20].

By (5.33), the hypersurface $\partial M \subset\left(\mathbb{R}^{3}, e\right)$, and further $\Theta=0$ on $\partial M$. Therefore $\partial M$ equals a plane in $\left(\mathbb{R}^{3}, e\right)$. This shows that $(M, g) \simeq\left(\mathbb{H}^{+}, e\right)$ and in particular,

$$
\|(M, g)\|_{H^{2}, 1}^{b . h .}=0 .
$$


5.4. The contradiction. In this section, we conclude the proof of Theorem 5.1.

On the one hand, by Sections 5.1 and 5.2 , the sequence $\left(M_{i}, g_{i}, p_{i}\right)$ is such that

$$
\left\|\left(M_{i}, g_{i}, p_{i}\right)\right\|_{H^{2}, 1}^{b . h .} \geq Q / 2,
$$

and

$$
\left(M_{i}, g_{i}, p_{i}\right) \rightarrow(M, g, p) \text { as } i \rightarrow \infty \text { in the pointed } H^{2} \text {-topology. }
$$

By Proposition 4.12, this implies that

$$
\|(M, g, p)\|_{H^{2}, 1}^{b . h .} \geq Q / 2 \neq 0 .
$$

On the other hand, by Section $5.3,(M, g) \simeq\left(\mathbb{H}^{+}, e\right)$, so that

$$
\|(M, g)\|_{H^{2}, 1}^{b . h .}=0 .
$$

This contradiction finishes the proof of Theorem 5.1.

\section{Higher REgularity ESTimates FOR BOUNDARY HARMONIC COORDINATES}

The main result of this section is the following.

Proposition 6.1 (Higher regularity of boundary harmonic coordinates). Let $g$ be a Riemannian metric in boundary harmonic coordinates on $B^{+}(x, r)$ such that for some $\varepsilon_{0}>0$,

$$
\|g-e\|_{\mathcal{H}^{2}\left(B^{+}(x, r)\right)}<\varepsilon_{0} .
$$

Assume in addition that for an integer $m \geq 0$,

$$
\sum_{i=0}^{m}\left\|\nabla^{(i)} \operatorname{Ric}\right\|_{\mathcal{L}^{2}\left(B^{+}(x, r)\right)}<\infty .
$$

Then, there exists $\varepsilon>0$ such that if $\varepsilon_{0}<\varepsilon$, then for all reals $0<r^{\prime}<r$,

$$
\|g\|_{\mathcal{H}^{m+2}\left(B^{+}\left(x, r^{\prime}\right)\right)} \leq C_{r^{\prime}, r} \sum_{i=0}^{m}\left\|\nabla^{(i)} \operatorname{Ric}\right\|_{\mathcal{L}^{2}\left(B^{+}(x, r)\right)}+C_{r^{\prime}, r, m}\|g\|_{\mathcal{H}^{2}\left(B^{+}(x, r)\right)} .
$$

Before proving Proposition 6.1, we first prove the higher regularity estimates of Theorem 3.1.

Proof of part (2) of Theorem 3.1. First, by Theorem 5.1, there is a real

$$
r_{0}=r_{0}\left(\|\operatorname{Ric}\|_{L^{2}(M)},\|\Theta\|_{L^{4}(\partial M)}, r_{v o l}(M, 1), \varepsilon / 2\right)>0
$$

such that for each $p \in M$ there exists a boundary harmonic chart $\varphi: B^{+}\left(x, r_{0}\right) \rightarrow U \subset M$ with $\varphi(x)=p$ such that on $B^{+}\left(x, r_{0}\right)$,

$$
(1-\varepsilon / 2) e_{i j} \leq g_{i j} \leq(1+\varepsilon / 2) e_{i j}
$$

and

$$
\left.r_{0}^{-1 / 2}\|\partial g\|_{\mathcal{L}^{2}\left(B^{+}\left(x, r_{0}\right)\right)}+r_{0}^{1 / 2}\left\|\partial^{2} g\right\|_{\mathcal{L}^{2}\left(B^{+}\left(x, r_{0}\right)\right)}\right) \leq \varepsilon / 2
$$


Therefore, by restriction, it holds in particular on $B^{+}\left(x, r_{0} / 2\right)$

$$
(1-\varepsilon) e_{i j} \leq g_{i j} \leq(1+\varepsilon) e_{i j}
$$

and

$$
\left(\frac{r_{0}}{2}\right)^{-1 / 2}\|\partial g\|_{\mathcal{L}^{2}\left(B^{+}\left(x, r_{0} / 2\right)\right)}+\left(\frac{r_{0}}{2}\right)^{1 / 2}\left\|\partial^{2} g\right\|_{\mathcal{L}^{2}\left(B^{+}\left(x, r_{0} / 2\right)\right)} \leq \varepsilon
$$

Second, by Proposition 6.1,

$$
\begin{aligned}
\|g\|_{\mathcal{H}^{m+2}\left(B^{+}\left(x, r_{0} / 2\right)\right)} & \leq C_{r_{0}} \sum_{i=0}^{m}\left\|\nabla^{(i)} \operatorname{Ric}\right\|_{\mathcal{L}^{2}\left(B^{+}\left(x, r_{0}\right)\right)}+C_{r_{0}, m}\|g\|_{\mathcal{H}^{2}\left(B^{+}\left(x, r_{0}\right)\right)} \\
& \leq C_{r_{0}} \sum_{i=0}^{m}\left\|\nabla^{(i)} \operatorname{Ric}\right\|_{\mathcal{L}^{2}(M)}+C_{r_{0}, m} \varepsilon
\end{aligned}
$$

Setting $r:=\frac{r_{0}}{2}$, it follows that (6.1), (6.2) and (6.3) prove part (2) of Theorem 3.1.

We turn now to the proof of Proposition 6.1. The higher regularity estimates are derived by applying standard elliptic estimates to the equations (see Lemma 2.15)

$$
\begin{aligned}
\frac{1}{2} \triangle_{g} g_{A B}+Q_{A B} & =-2 K & & \text { on } \underline{B}^{+}(x, r), \\
\frac{1}{2} \triangle g_{A B}+Q_{A B} & =-\operatorname{Ric}_{A B} & & \text { on } B^{+}(x, r), \\
\frac{1}{2} \triangle g^{3 j}+Q^{3 j} & =\operatorname{Ric}^{3 j} & & \text { on } B^{+}(x, r) .
\end{aligned}
$$

For completeness, we outline the proof of Proposition 6.1 in the rest of this section. The proof of Proposition 6.1 is based on induction.

- The induction basis. The case $m=0$ is direct.

- The induction step. Let $m \geq 1$. It suffices to prove that if $\varepsilon>0$ is sufficiently small, then for $0<r^{\prime}<r$,

$$
\begin{aligned}
\left\|\partial^{m+2} g\right\|_{L^{2}\left(B^{+}\left(x, r^{\prime}\right)\right)} \leq & C_{r^{\prime}, r}\left(\left\|\nabla^{(m)} \operatorname{Ric}\right\|_{L^{2}\left(B^{+}(x, r)\right)}+\|g\|_{\mathcal{H}^{m+1}\left(B^{+}(x, r)\right)}\right) \\
& +C_{r^{\prime}, r, m}\|g-e\|_{\mathcal{H}^{2}\left(B^{+}(x, r)\right)} .
\end{aligned}
$$

In the following, we prove (6.7) by using standard elliptic estimates. Let $\chi:=\chi_{x, r^{\prime}, r}$ be the smooth cut-off function defined in (2.4). For ease of notation, the constant in $\lesssim$ is here allowed to depend on $r^{\prime}$ and $r$, and we tactily use Lemma 2.7 and standard product estimates as in Lemma 2.5. We assume throughout the proof that $\varepsilon>0$ is sufficiently small. 
Control of $g_{A B}$. By Theorem B.4 applied to (6.4), we have

$$
\begin{aligned}
& \left\|\not \partial^{m-1} \not \phi_{A B}\right\|_{H^{5 / 2}\left(\underline{B}^{+}\left(x, r^{\prime}\right)\right)} \\
& \leq\left\|\not \partial^{m-1}\left(\chi \not \phi_{A B}\right)\right\|_{H^{5 / 2}\left(\underline{B}^{+}(x, r)\right)} \\
& \lesssim\left\|\mathcal{A}_{g}\left(\not \partial^{m-1}\left(\chi \not_{A B}\right)\right)\right\|_{H^{1 / 2}\left(\underline{B}^{+}(x, r)\right)} \\
& \lesssim\left\|\chi \not^{m-1}\left(\not_{\not g} \phi_{A B}\right)\right\|_{H^{1 / 2}\left(\underline{B}^{+}(x, r)\right)}+\|\not g-e\|_{\mathcal{H}^{3 / 2}\left(\underline{B}^{+}(x, r)\right)}\left\|\not \partial^{m-1}(\chi \not \phi)\right\|_{\mathcal{H}^{3 / 2}\left(\underline{B}^{+}\right)} \\
& +\|\not\|_{\mathcal{H}^{m+1 / 2}\left(\underline{B}^{+}(x, r)\right)}+C_{m}\|\not g-e\|_{\mathcal{H}^{3 / 2}\left(\underline{B}^{+}(x, r)\right)},
\end{aligned}
$$

where we estimated

$$
\begin{aligned}
& \left\|\left[\not_{\not g}, \not \partial^{m-1}\right]\left(\chi \not \phi_{A B}\right)\right\|_{H^{1 / 2}\left(\underline{B}^{+}(x, r)\right)}=\left\|\left[\not \phi^{C D} \not \partial_{C} \not D_{D}, \not^{m-1}\right]\left(\chi \not \phi_{A B}\right)\right\|_{H^{1 / 2}\left(\underline{B}^{+}(x, r)\right)} \\
& \lesssim\|\not y-e\|_{\mathcal{H}^{3 / 2}\left(\underline{B}^{+}(x, r)\right)}\left\|\not \partial^{m-1}(\chi \not \mathcal{H})\right\|_{\mathcal{H}^{3 / 2}\left(\underline{B}^{+}(x, r)\right)} \\
& +\|\not g\|_{\mathcal{H}^{m+1 / 2}\left(\underline{B}^{+}(x, r)\right)}+C_{m}\|\not g-e\|_{\mathcal{H}^{3 / 2}\left(\underline{B}^{+}(x, r)\right)} \text {. }
\end{aligned}
$$

The first term on the right-hand side of (6.8) is estimated by using (6.4) and the twice traced Gauss equation $2 K=\mathrm{R}_{\text {scal }}-2 \operatorname{Ric}(N, N)+(\operatorname{tr} \Theta)^{2}-|\Theta|^{2}$,

$$
\begin{aligned}
& \left\|\chi \not \not^{m-1}\left(\chi_{g} \not_{A B}\right)\right\|_{H^{1 / 2}\left(\underline{B}^{+}(x, r)\right)} \\
\leq & \left\|\chi \not \not^{m-1} \phi_{A B}\right\|_{H^{1 / 2}\left(\underline{B}^{+}(x, r)\right)}+\left\|\chi \not \not^{m-1}\left(\mathrm{R}_{s c a l}-2 \operatorname{Ric}_{N N}\right)\right\|_{H^{1 / 2}\left(\underline{B}^{+}(x, r)\right)} \\
& +\left\|\chi \not \partial^{m-1}\left((\operatorname{tr} \Theta)^{2}-|\Theta|^{2}\right)\right\|_{H^{1 / 2}\left(\underline{B}^{+}(x, r)\right)} \\
\lesssim & \left\|\nabla^{(m)} \operatorname{Ric}\right\|_{\mathcal{L}^{2}\left(B^{+}(x, r)\right)}+\|g-e\|_{\mathcal{H}^{2}\left(B^{+}(x, r)\right)}\left\|\partial^{m+2}(\chi g)\right\|_{\mathcal{L}^{2}\left(B^{+}(x, r)\right)} \\
& +\|g\|_{\mathcal{H}^{m+1}\left(B^{+}(x, r)\right)}+C_{m}\|g-e\|_{\mathcal{H}^{2}\left(B^{+}(x, r)\right)} .
\end{aligned}
$$

Plugging (6.9) into (6.8) yields

$$
\begin{aligned}
& \left\|\not \partial^{m-1}\left(\chi \phi_{A B}\right)\right\|_{\mathcal{H}^{5 / 2}\left(\underline{B}^{+}(x, r)\right)} \\
& \lesssim\left\|\nabla^{(m)} \operatorname{Ric}\right\|_{\mathcal{L}^{2}\left(B^{+}(x, r)\right)}+\|g-e\|_{\mathcal{H}^{2}\left(B^{+}(x, r)\right)}\left\|\partial^{m+2}(\chi g)\right\|_{\mathcal{L}^{2}\left(B^{+}(x, r)\right)} \\
& \quad+\|g\|_{\mathcal{H}^{m+1}\left(B^{+}(x, r)\right)}+C_{m}\|g-e\|_{\mathcal{H}^{2}\left(B^{+}(x, r)\right)} .
\end{aligned}
$$

Control of $g_{A B}$. By a standard application of Theorem A.1 applied to (6.5), we have

$$
\begin{aligned}
& \left\|\partial^{m} g_{A B}\right\|_{H^{2}\left(B^{+}\left(x, r^{\prime}\right)\right)} \\
\leq & \left\|\partial^{m}\left(\chi g_{A B}\right)\right\|_{H^{2}\left(B^{+}(x, r)\right)} \\
\lesssim & \left\|\chi \partial^{m}\left(\triangle_{g} g_{A B}\right)\right\|_{L^{2}\left(B^{+}(x, r)\right)}+\left\|\partial^{m}\left(\chi g_{A B}\right)\right\|_{H^{3 / 2}\left(\underline{B}^{+}(x, r)\right)} \\
& +\|g-e\|_{\mathcal{H}^{2}\left(B^{+}(x, r)\right)}\left\|\partial^{m+2}(\chi g)\right\|_{\mathcal{L}^{2}\left(B^{+}(x, r)\right)} \\
& +\|g\|_{\mathcal{H}^{m+1}\left(B^{+}(x, r)\right)}+C_{m}\|g-e\|_{\mathcal{H}^{2}\left(B^{+}(x, r)\right)},
\end{aligned}
$$

where we estimated

$$
\begin{aligned}
\left\|\left[\triangle_{g}, \partial^{m}\right]\left(\chi g_{A B}\right)\right\|_{H^{3 / 2}\left(\underline{B}^{+}(x, r)\right)} \lesssim & \|g-e\|_{\mathcal{H}^{2}\left(B^{+}(x, r)\right)}\left\|\partial^{m+2}(\chi g)\right\|_{\mathcal{L}^{2}\left(B^{+}(x, r)\right)} \\
& +\|g\|_{\mathcal{H}^{m+1}\left(B^{+}(x, r)\right)}+C_{m}\|g-e\|_{\mathcal{H}^{2}\left(B^{+}(x, r)\right)} .
\end{aligned}
$$


The first term on the right-hand side of (6.11) is estimated as above by using (6.5),

$$
\begin{aligned}
& \left\|\chi \partial^{m}\left(\triangle_{g} g_{A B}\right)\right\|_{L^{2}\left(B^{+}(x, r)\right)} \\
\lesssim & \left\|\chi \partial^{m} \operatorname{Ric}\right\|_{\mathcal{L}^{2}\left(B^{+}(x, r)\right)}+\left\|\chi \partial^{m} Q_{A B}\right\|_{L^{2}\left(B^{+}(x, r)\right)} \\
\lesssim & \left\|\nabla^{(m)} \operatorname{Ric}\right\|_{\mathcal{L}^{2}\left(B^{+}(x, r)\right)}+\|g-e\|_{\mathcal{H}^{2}\left(B^{+}(x, r)\right)}\left\|\partial^{m+2}(\chi g)\right\|_{L^{2}\left(B^{+}(x, r)\right)} \\
& +\|g\|_{\mathcal{H}^{m+1}\left(B^{+}(x, r)\right)}+C_{m}\|g-e\|_{\mathcal{H}^{2}\left(B^{+}(x, r)\right)} .
\end{aligned}
$$

The second term on the right-hand side of (6.11) is estimated by using that $g_{A B}=\phi_{A B}$ on $\underline{B}^{+}(x, r)$ and (6.10), yielding

$$
\begin{aligned}
& \left\|\not \partial^{m}\left(\chi g_{A B}\right)\right\|_{H^{3 / 2}\left(\underline{B}^{+}(x, r)\right)} \\
= & \left\|\partial^{m}\left(\chi \phi_{A B}\right)\right\|_{H^{3 / 2}\left(\underline{B}^{+}(x, r)\right)} \\
\lesssim & \left\|\nabla^{(m)} \operatorname{Ric}\right\|_{\mathcal{L}^{2}\left(B^{+}(x, r)\right)}+\|g-e\|_{\mathcal{H}^{2}\left(B^{+}(x, r)\right)}\left\|\partial^{m+2}(\chi g)\right\|_{\mathcal{L}^{2}\left(B^{+}(x, r)\right)} \\
& +\|g\|_{\mathcal{H}^{m+1}\left(B^{+}(x, r)\right)}+C_{m}\|g-e\|_{\mathcal{H}^{2}\left(B^{+}(x, r)\right)} .
\end{aligned}
$$

Plugging (6.12) and (6.13) into (6.11) yields

$$
\begin{aligned}
& \left\|\partial^{m}\left(\chi g_{A B}\right)\right\|_{H^{2}\left(B^{+}(x, r)\right)} \\
& \lesssim\left\|\nabla^{(m)} \operatorname{Ric}\right\|_{\mathcal{L}^{2}\left(B^{+}(x, r)\right)}+\|g-e\|_{\mathcal{H}^{2}\left(B^{+}(x, r)\right)}\left\|\partial^{m+2}(\chi g)\right\|_{\mathcal{L}^{2}\left(B^{+}(x, r)\right)} \\
& \quad+\|g\|_{\mathcal{H}^{m+1}\left(B^{+}(x, r)\right)}+C_{m}\|g-e\|_{\mathcal{H}^{2}\left(B^{+}(x, r)\right)} .
\end{aligned}
$$

In particular, (6.14) implies by $\left.\Theta_{A B}=\frac{1}{2 a} \partial_{3}\left(g_{A B}\right)-\frac{1}{2 a}\left(\mathcal{A}_{\beta} \not\right)\right)_{A B}$, see $(2.8)$, that

$$
\begin{aligned}
& \left\|\chi \not \partial^{m} \operatorname{tr} \Theta\right\|_{H^{1 / 2}\left(\underline{B}^{+}(x, r)\right)} \\
& \lesssim\left\|\nabla^{(m)} \operatorname{Ric}\right\|_{\mathcal{L}^{2}\left(B^{+}(x, r)\right)}+\|g-e\|_{\mathcal{H}^{2}\left(B^{+}(x, r)\right)}\left\|\partial^{m+2}(\chi g)\right\|_{\mathcal{L}^{2}\left(B^{+}(x, r)\right)} \\
& \quad+\|g\|_{\mathcal{H}^{m+1}\left(B^{+}(x, r)\right)}+C_{m}\|g-e\|_{\mathcal{H}^{2}\left(B^{+}(x, r)\right)} .
\end{aligned}
$$

Control of $g^{33}$. By a standard application of Theorem A.6 to (6.6), we have

$$
\begin{aligned}
& \left\|\partial^{m} g^{33}\right\|_{H^{2}\left(B^{+}\left(x, r^{\prime}\right)\right)} \\
\leq & \left\|\partial^{m}\left(\chi g^{33}\right)\right\|_{H^{2}\left(B^{+}(x, r)\right)} \\
\lesssim & \left\|\chi \partial^{m}\left(\triangle_{g} g^{33}\right)\right\|_{L^{2}\left(B^{+}(x, r)\right)}+\left\|\chi \not^{m} N\left(g^{33}\right)\right\|_{H^{1 / 2}\left(\underline{B}^{+}(x, r)\right)} \\
& +\|g-e\|_{\mathcal{H}^{2}\left(B^{+}(x, r)\right)}\left\|\partial^{m+2}(\chi g)\right\|_{\mathcal{L}^{2}\left(B^{+}(x, r)\right)} \\
& +\|g\|_{\mathcal{H}^{m+1}\left(B^{+}(x, r)\right)}+C_{m}\|g-e\|_{\mathcal{H}^{2}\left(B^{+}(x, r)\right)}
\end{aligned}
$$

The first term on the right-hand side of (6.16) is estimated by using (6.6),

$$
\begin{aligned}
& \quad\left\|\chi \partial^{m}\left(\triangle_{g} g^{33}\right)\right\|_{L^{2}\left(B^{+}(x, r)\right)} \\
& \lesssim\left\|\nabla^{(m)} \operatorname{Ric}\right\|_{\mathcal{L}^{2}\left(B^{+}(x, r)\right)}+\|g-e\|_{\mathcal{H}^{2}\left(B^{+}(x, r)\right)}\left\|\partial^{m+2}(\chi g)\right\|_{\mathcal{L}^{2}\left(B^{+}(x, r)\right)} \\
& \quad+\|g\|_{\mathcal{H}^{m+1}\left(B^{+}(x, r)\right)}+C_{m}\|g-e\|_{\mathcal{H}^{2}\left(B^{+}(x, r)\right)} .
\end{aligned}
$$

The second term on the right-hand side of (6.16) is estimated by using that

$$
N\left(g^{33}\right)=2 g^{33} \operatorname{tr} \Theta
$$


in boundary harmonic coordinates, see Lemma 2.17, together with the previous estimate (6.15),

$$
\begin{aligned}
& \left\|\chi \not \not^{m} N\left(g^{33}\right)\right\|_{H^{1 / 2}\left(\underline{B}^{+}(x, r)\right)} \\
= & \left\|\chi \not \partial \partial^{m}\left(2 g^{33} \operatorname{tr} \Theta\right)\right\|_{H^{1 / 2}\left(\underline{B}^{+}(x, r)\right)} \\
\lesssim & \left\|\nabla^{(m)} \operatorname{Ric}\right\|_{\mathcal{L}^{2}\left(B^{+}(x, r)\right)}+\|g-e\|_{\mathcal{H}^{2}\left(B^{+}(x, r)\right)}\left\|\partial^{m+2}(\chi g)\right\|_{\mathcal{L}^{2}\left(B^{+}(x, r)\right)} \\
& +\|g\|_{\mathcal{H}^{m+1}\left(B^{+}(x, r)\right)}+C_{m}\|g-e\|_{\mathcal{H}^{2}\left(B^{+}(x, r)\right)} .
\end{aligned}
$$

Plugging (6.17) and (6.18) into (6.16) yields

$$
\begin{aligned}
\left\|\partial^{m-1}\left(\chi g^{33}\right)\right\|_{W^{2,3}\left(B^{+}(x, r)\right) \lesssim} & \|g-e\|_{\mathcal{H}^{2}\left(B^{+}(x, r)\right)}\left\|\partial^{m+2}(\chi g)\right\|_{\mathcal{L}^{2}\left(B^{+}(x, r)\right)} \\
& +\left\|\chi \partial^{m} \operatorname{Ric}\right\|_{\mathcal{L}^{2}\left(B^{+}(x, r)\right)}+\|g\|_{\mathcal{H}^{m+1}\left(B^{+}(x, r)\right)} \\
& +C_{m}\|g-e\|_{\mathcal{H}^{2}\left(B^{+}(x, r)\right)} .
\end{aligned}
$$

Control of $g^{3 A}$. By a standard application of Theorem A.6 to (6.6), we have

$$
\begin{aligned}
& \left\|\partial^{m} g^{3 A}\right\|_{H^{2}\left(B^{+}\left(x, r^{\prime}\right)\right)} \\
\leq & \left\|\partial^{m}\left(\chi g^{3 A}\right)\right\|_{H^{2}\left(B^{+}(x, r)\right)} \\
\lesssim & \left\|\chi \partial^{m}\left(\triangle_{g} g^{3 A}\right)\right\|_{L^{2}\left(B^{+}(x, r)\right)}+\left\|\chi \not \partial^{m} N\left(g^{3 A}\right)\right\|_{H^{1 / 2}\left(\underline{B}^{+}(x, r)\right)} \\
& +\|g-e\|_{\mathcal{H}^{2}\left(B^{+}(x, r)\right)}\left\|\partial^{m+2}(\chi g)\right\|_{\mathcal{L}^{2}\left(B^{+}(x, r)\right)} \\
& +\|g\|_{\mathcal{H}^{m+1}\left(B^{+}(x, r)\right)}+C_{m}\|g-e\|_{\mathcal{H}^{2}\left(B^{+}(x, r)\right)} .
\end{aligned}
$$

The first term on the right-hand side of (6.20) is estimated by using (6.6),

$$
\begin{aligned}
& \quad\left\|\chi \partial^{m}\left(\triangle_{g} g^{3 A}\right)\right\|_{L^{2}\left(B^{+}(x, r)\right)} \\
& \lesssim\left\|\nabla^{(m)} \operatorname{Ric}\right\|_{\mathcal{L}^{2}\left(B^{+}(x, r)\right)}+\|g-e\|_{\mathcal{H}^{2}\left(B^{+}(x, r)\right)}\left\|\partial^{m+2}(\chi g)\right\|_{\mathcal{L}^{2}\left(B^{+}(x, r)\right)} \\
& \quad+\|g\|_{\mathcal{H}^{m+1}\left(B^{+}(x, r)\right)}+C_{m}\|g-e\|_{\mathcal{H}^{2}\left(B^{+}(x, r)\right)} .
\end{aligned}
$$

The second term on the right-hand side of (6.20) is estimated by using that

$$
N\left(g^{3 A}\right)=g^{3 A} \operatorname{tr} \Theta-\frac{1}{2} \frac{1}{\sqrt{g^{33}}} g^{3 i} \partial_{i} g^{33},
$$

in boundary harmonic coordinates, see Lemma 2.17, together with (6.15) and (6.19),

$$
\begin{aligned}
& \left\|\chi \not \not^{m} N\left(g^{33}\right)\right\|_{H^{1 / 2}\left(\underline{B}^{+}(x, r)\right)} \\
= & \left\|\chi \not \not^{m}\left(2 g^{33} \operatorname{tr} \Theta\right)\right\|_{H^{1 / 2}\left(\underline{B}^{+}(x, r)\right)} \\
\lesssim & \left\|\nabla^{(m)} \operatorname{Ric}\right\|_{\mathcal{L}^{2}\left(B^{+}(x, r)\right)}+\|g-e\|_{\mathcal{H}^{2}\left(B^{+}(x, r)\right)}\left\|\partial^{m+2}(\chi g)\right\|_{\mathcal{L}^{2}\left(B^{+}(x, r)\right)} \\
& +\|g\|_{\mathcal{H}^{m+1}\left(B^{+}(x, r)\right)}+C_{m}\|g-e\|_{\mathcal{H}^{2}\left(B^{+}(x, r)\right)} .
\end{aligned}
$$


Plugging (6.21) and (6.22) into (6.20) yields

$$
\begin{aligned}
& \quad\left\|\partial^{m}\left(\chi g^{3 A}\right)\right\|_{H^{2}\left(B^{+}(x, r)\right)} \\
& \lesssim\left\|\nabla^{(m)} \operatorname{Ric}\right\|_{\mathcal{L}^{2}\left(B^{+}(x, r)\right)}+\|g-e\|_{\mathcal{H}^{2}\left(B^{+}(x, r)\right)}\left\|\partial^{m+2}(\chi g)\right\|_{\mathcal{L}^{2}\left(B^{+}(x, r)\right)} \\
& \quad+\|g\|_{\mathcal{H}^{m+1}\left(B^{+}(x, r)\right)}+C_{m}\|g-e\|_{\mathcal{H}^{2}\left(B^{+}(x, r)\right)} .
\end{aligned}
$$

Conclusion of the proof of (6.7). By Lemma 2.18, the estimates (6.10), (6.14), (6.19) and (6.23) imply that

$$
\begin{aligned}
& \quad\left\|\partial^{m}(\chi g)\right\|_{\mathcal{H}^{2}\left(B^{+}(x, r)\right)} \\
& \lesssim\left\|\nabla^{(m)} \operatorname{Ric}\right\|_{\mathcal{L}^{2}\left(B^{+}(x, r)\right)}+\|g-e\|_{\mathcal{H}^{2}\left(B^{+}(x, r)\right)}\left\|\partial^{m+2}(\chi g)\right\|_{\mathcal{L}^{2}\left(B^{+}(x, r)\right)} \\
& \quad+\|g\|_{\mathcal{H}^{m+1}\left(B^{+}(x, r)\right)}+C_{m}\|g-e\|_{\mathcal{H}^{2}\left(B^{+}(x, r)\right)} \\
& \lesssim\left\|\nabla^{(m)} \operatorname{Ric}\right\|_{\mathcal{L}^{2}\left(B^{+}(x, r)\right)}+\|g\|_{\mathcal{H}^{m+1}\left(B^{+}(x, r)\right)}+C_{m}\|g-e\|_{\mathcal{H}^{2}\left(B^{+}(x, r)\right)},
\end{aligned}
$$

where we used that $\varepsilon>0$ is sufficiently small to absorb the second term in the left-hand side. This finishes the proof of (6.7) and concludes the induction step. This finishes the proof of Proposition 6.1.

\section{Appendix A. Global elliptic estimates}

In this section, we collect global elliptic estimates for Dirichlet and Neumann problems, see Sections A.1 and A.2, respectively. These estimates are applied in Section 4 to construct boundary harmonic coordinates, and in Section 6 to derive higher regularity estimates for given boundary harmonic coordinates.

The estimates are standard, see for example [9] or [20], but we give some proofs for completeness. In the following, the constant in $\lesssim$ is allowed to depend on the domain $\Omega$.

A.1. Global estimates for Dirichlet data. We have the following standard elliptic estimates, see for example Theorem 9.13 from [9] and Lemma 2.4.

Theorem A.1 (Standard global elliptic estimate). Let $n \in\{2,3\}$ and let $\Omega \subset \mathbb{R}^{n}$ be a bounded smooth domain. For a real $n<p<\infty$, let $g \in \mathcal{W}^{1, p}(\Omega)$ be a Riemannian metric such that $\triangle_{g}=g^{i j} \partial_{i} \partial_{j}$. Then, for all $1<p^{\prime}<\infty$ and $u \in W^{2, p^{\prime}}(\Omega)$, we have

$$
\|u\|_{W^{2, p^{\prime}}(\Omega)} \lesssim\left\|\triangle_{g} u\right\|_{L^{p^{\prime}(\Omega)}}+\|u\|_{W^{2-1 / p^{\prime}, p^{\prime}(\partial \Omega)}} .
$$

From Theorem A.1 we get the following standard higher regularity estimate, see for example Theorem 8.13 in [9].

Corollary A.2 (Standard global higher regularity elliptic estimate). Let $\Omega \subset \mathbb{R}^{3}$ be a bounded smooth domain. Let the Riemannian metric $g \in \mathcal{H}^{2}(\Omega)$ be such that $\triangle_{g}=g^{i j} \partial_{i} \partial_{j}$. Then for all $u \in H^{3}(\Omega)$,

$$
\|u\|_{H^{3}(\Omega)} \lesssim\left\|\triangle_{g} u\right\|_{H^{1}(\Omega)}+\|u\|_{H^{5 / 2}(\partial \Omega)} .
$$


We now turn to the Laplace-Beltrami operator of a general Riemannian metric in three dimensions.

Proposition A.3. Let $\Omega \subset \mathbb{R}^{3}$ be bounded smooth domain and $g \in \mathcal{W}^{1,6}(\Omega)$ a Riemannian metric. Let $u$ be the solution to

$$
\begin{aligned}
\triangle_{g} u & =f \text { in } \Omega, \\
u & =0 \text { on } \partial \Omega .
\end{aligned}
$$

for $f \in L^{6}$. Then $u \in W^{2,6}(\Omega)$ and

$$
\|u\|_{W^{2,6}(\Omega)} \lesssim\|f\|_{L^{6}(\Omega)} .
$$

Proof. First, we note that by the Lax-Milgram theorem and Lemma 2.4, there exists a solution $u \in H^{1}(\Omega)$ with

$$
\|u\|_{H^{1}(\Omega)} \lesssim\|f\|_{H^{-1}(\Omega)} \lesssim\|f\|_{L^{6}(\Omega)}
$$

and consequently also

$$
\|u\|_{L^{6}(\Omega)} \lesssim\|f\|_{L^{6}(\Omega)}
$$

By Theorem A.1,

$$
\begin{aligned}
\|u\|_{W^{2,6}(\Omega)} & \lesssim\left\|g^{i j} \partial_{i} \partial_{j} u\right\|_{L^{6}(\Omega)} \\
& \lesssim\|f\|_{L^{6}(\Omega)}+\left\|\triangle_{g} u-g^{i j} \partial_{i} \partial_{j} u\right\|_{L^{6}(\Omega)} \\
& \lesssim\|f\|_{L^{6}(\Omega)}+\|\partial g \partial u\|_{L^{6}(\Omega)} \\
& \lesssim\|f\|_{L^{6}(\Omega)}+\|g\|_{\mathcal{W}^{1,6}(\Omega)}\|\partial u\|_{C^{0}(\Omega)} .
\end{aligned}
$$

In the following, we control the term $\|\partial u\|_{C^{0}(\Omega)}$ on the right-hand side of (A.3) by a bootstrap argument ${ }^{7}$. Using that $g \in \mathcal{W}^{1,6}(\Omega) \subset \mathcal{C}^{0}(\Omega)$ in $n=3$, we have by partial integration on $\Omega$,

$$
\|\partial u\|_{L^{2}(\Omega)} \lesssim\|\nabla u\|_{L^{2}(\Omega)} \lesssim\|u\|_{L^{2}(\Omega)}^{1 / 2}\left\|\triangle_{g} u\right\|_{L^{2}(\Omega)}^{1 / 2} \lesssim\|f\|_{L^{6}(\Omega)} .
$$

By using Theorem A.1 with $p^{\prime}=3 / 2$, this implies further

$$
\begin{aligned}
\|u\|_{W^{2,3 / 2}(\Omega)} & \lesssim\left\|g^{i j} \partial_{i} \partial_{j} u\right\|_{L^{3 / 2}(\Omega)} \\
& \lesssim\left\|\triangle_{g} u\right\|_{L^{3 / 2}(\Omega)}+\|\partial g \partial u\|_{L^{3 / 2}(\Omega)} \\
& \lesssim\|f\|_{L^{3 / 2}(\Omega)}+\|g\|_{\mathcal{W}^{1,6}(\Omega)}\|\partial u\|_{L^{2}(\Omega)} \\
& \lesssim\|f\|_{L^{3 / 2}(\Omega)}+\|g\|_{\mathcal{W}^{1,6}(\Omega)}\|f\|_{L^{6}(\Omega)}
\end{aligned}
$$

By Lemma 2.4 and (A.5), we have

$$
\begin{aligned}
\|\partial u\|_{L^{3}(\Omega)} & \lesssim\|\partial u\|_{W^{1,3 / 2}(\Omega)} \\
& \lesssim\|f\|_{3 / 2(\Omega)}+\|g\|_{\mathcal{W}^{1,6}(\Omega)}\|f\|_{L^{6}(\Omega)} \\
& \lesssim\|f\|_{L^{6}(\Omega)} .
\end{aligned}
$$

\footnotetext{
${ }^{7}$ This argument is taken from [20].
} 
Compared to (A.4), we bootstrapped the regularity of $\partial u$.

By continuing to bootstrap the regularity of $\partial u$ with Theorem A.1 and Lemma 2.4 as above (with $p^{\prime}=2, p^{\prime}=3$ and finally $p^{\prime}=24 / 5$ ), we get

$$
\|\partial u\|_{W^{1,24 / 5}(\Omega)} \lesssim\|f\|_{L^{6}(\Omega)},
$$

and consequently, by Lemma 2.4 and (A.2),

$$
\begin{aligned}
\|\partial u\|_{C^{0}(\Omega)} & \lesssim\|\partial u\|_{W^{1,24 / 5}(\Omega)} \\
& \lesssim\|f\|_{L^{6}(\Omega)} .
\end{aligned}
$$

Plugging this bound into (A.3) proves (A.1). This finishes the proof of Proposition A.3.

Corollary A.4 (Global estimates for the Dirichlet problem in $H^{3}, n=3$ ). Let $\Omega \subset \mathbb{R}^{3}$ be bounded smooth domain and $g \in \mathcal{H}^{2}(\Omega)$ a Riemannian metric. Let $u$ be the solution to

$$
\begin{aligned}
\triangle_{g} u & =f \text { in } \Omega, \\
u & =h \text { on } \partial \Omega .
\end{aligned}
$$

for $f \in H^{1}(\Omega)$ and $h \in H^{5 / 2}(\partial \Omega)$. Then we have $u \in H^{3}(\Omega)$ and

$$
\|u\|_{H^{3}(\Omega)} \lesssim\|f\|_{H^{1}(\Omega)}+\|h\|_{H^{5 / 2}(\partial \Omega)} .
$$

Proof. First, we reduce to homogeneous Dirichlet data. By Paragraph 7.41 in [1], there exists $\tilde{h} \in H^{3}(\Omega)$, such that

$$
\tau(\tilde{h})=h \text { on } \partial \Omega
$$

and

$$
\|\tilde{h}\|_{H^{3}(\Omega)} \lesssim\|h\|_{H^{5 / 2}(\partial \Omega)} .
$$

Therefore we can reduce (A.6) to the study of

$$
\begin{aligned}
\triangle_{g} u & =\tilde{f} \text { in } \Omega, \\
u & =0 \text { on } \partial \Omega .
\end{aligned}
$$

where $\tilde{f}:=f+\triangle_{g} \tilde{h}$ is bounded by

$$
\begin{aligned}
\|\tilde{f}\|_{H^{1}(\Omega)} & \lesssim\|f\|_{H^{1}(\Omega)}+\left\|\triangle_{g} \tilde{h}\right\|_{H^{1}(\Omega)} \\
& \lesssim\|f\|_{H^{1}(\Omega)}+\left\|g \partial^{2} \tilde{h}\right\|_{H^{1}(\Omega)}+\|\partial g \partial \tilde{h}\|_{H^{1}(\Omega)} \\
& \lesssim\|f\|_{H^{1}(\Omega)}+\|g\|_{\mathcal{H}^{2}(\Omega)}\|\tilde{h}\|_{H^{3}(\Omega)} .
\end{aligned}
$$

By the Lax-Milgram theorem there exists a unique solution $u$ to (A.7) satisfying

$$
\|u\|_{H^{1}(\Omega)} \lesssim\|\tilde{f}\|_{L^{2}(\Omega)} .
$$

In particular, by Lemma 2.4,

$$
\|u\|_{L^{6}(\Omega)} \lesssim\|u\|_{H^{1}(\Omega)} \lesssim\|\tilde{f}\|_{L^{2}(\Omega)} .
$$


Applying Corollary A.2 and Proposition A.3 to (A.7), we get

$$
\begin{aligned}
\|u\|_{H^{3}(\Omega)} & \lesssim\left\|g^{i j} \partial_{i} \partial_{j} u\right\|_{H^{1}(\Omega)}+\|u\|_{H^{5 / 2}(\partial \Omega)} \\
& \lesssim\left\|\triangle_{g} u\right\|_{H^{1}(\Omega)}+\|\partial g \partial u\|_{H^{1}(\Omega)} \\
& \lesssim\|\tilde{f}\|_{H^{1}(\Omega)}+\|g\|_{\mathcal{H}^{2}(\Omega)}\|\partial u\|_{C^{0}(\bar{\Omega})} \\
& \lesssim\|\tilde{f}\|_{H^{1}(\Omega)}+\|g\|_{\mathcal{H}^{2}(\Omega)}\|u\|_{W^{1,6}(\Omega)} \\
& \lesssim\|\tilde{f}\|_{H^{1}(\Omega)}+\|g\|_{\mathcal{H}^{2}(\Omega)}\|\tilde{f}\|_{L^{6}(\Omega)} \\
& \lesssim\|\tilde{f}\|_{H^{1}(\Omega)}, \\
& \lesssim\|f\|_{H^{1}(\Omega)}+\|h\|_{H^{5 / 2}(\partial \Omega)},
\end{aligned}
$$

where we used Lemma 2.4 and (A.8). This finishes the proof of Corollary A.4.

A.2. Global estimates for Neumann data. In this section we collect standard global elliptic estimates for given Neumann boundary data. These estimates are applied in Section 6 to the metric components $g^{33}$ and $g^{3 A}$ in boundary harmonic coordinates.

We recall the standard elliptic estimates in a smooth domain, see for example (2.3.3.1) in $[10]$.

Theorem A.5. Let $1<p<\infty$ be a real and $\Omega$ be a bounded smooth domain. Let $g \in$ $C^{0,1}(\bar{\Omega})$ be a Riemannian metric on $\bar{\Omega}$. Let further $B(u):=b^{i} \partial_{i} u$ be a boundary operator with $b^{i}$ Lipschitz on $\partial \Omega$ and $g(b, N) \geq c$ for some $c>0$. Then for any $u \in W^{2, p}(\Omega)$, we have

$$
\|u\|_{W^{2, p}(\Omega)} \lesssim\left\|\triangle_{g} u\right\|_{L^{p}(\Omega)}+\|B(u)\|_{W^{1-1 / p, p}(\partial \Omega)} .
$$

In the special case of boundary harmonic coordinates and Neumann boundary data, the proof of Theorem A.5 generalises to the following result.

Theorem A.6 (Global elliptic estimates for the Neumann problem, $n=3$ ). Let $3<$ $p<\infty$ be a real and let $\Omega \subset \mathbb{R}^{3}$ be a bounded smooth domain. Let $g \in \mathcal{W}^{1, p}(\Omega)$ be a Riemannian metric on $\Omega$ such that $\triangle_{g}=g^{i j} \partial_{i} \partial_{j}$. Then for every $1<p^{\prime}<\infty$ and $u \in W^{2, p^{\prime}}(\Omega)$,

$$
\|u\|_{W^{2, p^{\prime}}(\Omega)} \lesssim\left\|\triangle_{g} u\right\|_{L^{p^{\prime}}(\Omega)}+\|N(u)\|_{W^{1-1 / p^{\prime}, p^{\prime}(\partial \Omega)}} .
$$

Proof. The proof of Theorem A.5 in [10] uses first an analysis of the Neumann problem for a constant coefficient operator and second a continuity argument. We claim that both the same steps go through in the low regularity setting. Indeed, on the one hand, by Lemma 2.4 and $p>3$,

$$
g^{i j} \in W^{1, p} \subset C^{0, \alpha}
$$

for some $\alpha>0$. Therefore, at each point, $g^{i j} \partial_{i} \partial_{j}$ is a well-defined pointwise elliptic operator.

On the other hand, inspecting the continuity argument in the proof of Theorem A.5 in [10], it follows that in the case $\triangle_{g}=g^{i j} \partial_{i} \partial_{j}$ and $B=N$, the regularity $g^{i j} \in C^{0, \alpha}$ suffices 
to derive (A.9), see in particular the estimates (2.3.3.5) and (2.3.3.6) in [10] where the regularity of $g^{i j}$ is used. This finishes the proof of Theorem A.6.

\section{ApPENDiX B. INTERIOR ELLIPTIC ESTIMATES}

In this section we derive interior elliptic estimates in fractional regularity and control the Dirichlet problem for the Laplace-Beltrami operator.

We recall the following interior estimates, see Proposition 3.4 in [15].

Theorem B.1 (Interior estimates in $H^{3 / 2}$ ). Let $\Omega^{\prime} \subset \subset \Omega \subset \mathbb{R}^{2}$ be two bounded smooth domains, and let $g \in \mathcal{H}^{3 / 2}(\Omega)$ be a Riemannian metric. Then for all $u \in H^{3 / 2}(\Omega)$,

$$
\|u\|_{H^{3 / 2}\left(\Omega^{\prime}\right)} \lesssim\left\|\triangle_{g} u\right\|_{H^{-1 / 2}(\Omega)}+\|u\|_{H^{1 / 2}(\Omega)} .
$$

Moreover, we have the following interior estimates. They are derived analogously to Proposition A.3, see also Theorem A.3 in [20].

Proposition B.2 (Interior estimates in $\left.W^{2,4}\right)$. Let $\Omega^{\prime} \subset \subset \Omega \subset \mathbb{R}^{2}$ be two bounded smooth domains. Let $g \in \mathcal{W}^{1,4}(\Omega)$ be a Riemannian metric. Then for all $u \in W^{2,4}(\Omega)$,

$$
\|u\|_{W^{2,4}\left(\Omega^{\prime}\right)} \lesssim\left\|\triangle_{g} u\right\|_{L^{4}(\Omega)}+\|u\|_{L^{4}(\Omega)} .
$$

By combining Theorem B.1 and Proposition B.2, we have the following stronger interior elliptic estimates for the Laplace-Beltrami operator.

Corollary B.3 (Interior estimates in $H^{5 / 2}, n=2$ ). Let $\Omega^{\prime} \subset \subset \Omega \subset \mathbb{R}^{2}$ be two bounded smooth domains. Let $g \in \mathcal{H}^{3 / 2}(\Omega)$ be a Riemannian metric. Then for all $u \in H^{5 / 2}(\Omega)$, we have

$$
\|u\|_{H^{5 / 2}\left(\Omega^{\prime}\right)} \lesssim\left\|\triangle_{g} u\right\|_{H^{1 / 2}(\Omega)}+\|u\|_{H^{3 / 2}(\Omega)} .
$$

Proof. Let $g \in \mathcal{H}^{3 / 2}(\Omega)$ and let

$$
\Omega^{\prime} \subset \subset \Omega_{2} \subset \subset \Omega_{1} \subset \subset \Omega
$$

be bounded open subsets with smooth boundary. By Theorem B.1,

$$
\|u\|_{H^{3 / 2}\left(\Omega_{1}\right)} \lesssim\|\triangle u\|_{H^{-1 / 2}(\Omega)}+\|u\|_{H^{1 / 2}(\Omega)} .
$$

By Proposition B.2 and (B.2),

$$
\begin{aligned}
\|u\|_{W^{2,4}\left(\Omega_{2}\right)} & \lesssim\left\|\triangle_{g} u\right\|_{L^{4}\left(\Omega_{1}\right)}+\|u\|_{L^{4}\left(\Omega_{1}\right)} \\
& \lesssim\left\|\triangle_{g} u\right\|_{H^{1 / 2}(\Omega)}+\|u\|_{H^{1 / 2}(\Omega)} .
\end{aligned}
$$

Applying now Theorem B.1 to $\partial u$ and using (B.2) and (B.3), we get

$$
\begin{aligned}
\|\partial u\|_{H^{3 / 2}\left(\Omega^{\prime}\right)} & \lesssim\left\|\triangle_{g} \partial u\right\|_{H^{-1 / 2}\left(\Omega_{2}\right)}+\|\partial u\|_{H^{1 / 2}\left(\Omega_{2}\right)} \\
& \lesssim\left\|\triangle_{g} u\right\|_{H^{1 / 2}\left(\Omega_{2}\right)}+\left\|\left[\triangle_{g}, \partial\right] u\right\|_{H^{-1 / 2}\left(\Omega_{2}\right)}+\|u\|_{H^{3 / 2}\left(\Omega_{2}\right)} .
\end{aligned}
$$

The commutator term equals schematically

$$
\left[\triangle_{g}, \partial\right] u=g^{2} \partial^{2} g \partial u+g^{2} \partial g \partial^{2} u \text {. }
$$


By Lemma 2.4, the first term of the commutator is bounded by (B.3) as

$$
\begin{aligned}
\left\|g^{2} \partial^{2} g \partial u\right\|_{H^{-1 / 2}\left(\Omega_{2}\right)} & \lesssim\left\|\partial^{2} g \partial u\right\|_{H^{-1 / 2}\left(\Omega_{2}\right)} \\
& \lesssim\left\|\partial^{2} g\right\|_{\mathcal{H}^{-1 / 2}\left(\Omega_{2}\right)}\|\partial u\|_{W^{1,4}\left(\Omega_{2}\right)} \\
& \lesssim\|g\|_{\mathcal{H}^{3 / 2}\left(\Omega_{2}\right)}\|u\|_{W^{2,4}\left(\Omega_{2}\right)} \\
& \lesssim\|g\|_{\mathcal{H}^{3 / 2}(\Omega)}\left(\left\|\triangle_{g} u\right\|_{H^{1 / 2}(\Omega)}+\|u\|_{H^{1 / 2}(\Omega)}\right) .
\end{aligned}
$$

Similarly, the second term of the commutator is bounded by

$$
\begin{aligned}
\left\|g^{2} \partial g \partial^{2} u\right\|_{H^{-1 / 2}\left(\Omega_{2}\right)} & \lesssim\left\|\partial g \partial^{2} u\right\|_{H^{-1 / 2}\left(\Omega_{2}\right)} \\
& \lesssim\|\partial g\|_{\mathcal{H}^{1 / 2}\left(\Omega_{2}\right)}\left\|\partial^{2} u\right\|_{W^{1,4}\left(\Omega_{2}\right)} \\
& \lesssim\|g\|_{\mathcal{H}^{3 / 2}(\Omega)}\left(\left\|\triangle_{g} u\right\|_{H^{1 / 2}(\Omega)}+\|u\|_{H^{1 / 2}(\Omega)}\right) .
\end{aligned}
$$

Plugging this into (B.4) and summing over all coordinate derivatives proves (B.1). This finishes the proof of Proposition B.3.

The proof of the next corollary of Proposition B.3 follows by the Lax-Milgram theorem and is left to the reader.

Corollary B.4 (Interior estimates for the Dirichlet problem in $H^{5 / 2}, n=2$ ). Let $\Omega \subset \mathbb{R}^{2}$ be a bounded smooth domain and let $g \in H^{3 / 2}(\Omega)$ be a Riemannian metric on $\Omega$. Then for every $f \in H^{1 / 2}(\Omega)$, there exists a unique solution $u$ to

$$
\begin{aligned}
\triangle_{g} u & =f \text { in } \Omega, \\
u & =0 \text { on } \partial \Omega .
\end{aligned}
$$

Moreover, for every smooth domain $\Omega^{\prime} \subset \subset \Omega$, we have $u \in H^{5 / 2}\left(\Omega^{\prime}\right)$ and

$$
\|u\|_{H^{5 / 2}\left(\Omega^{\prime}\right)} \lesssim\|f\|_{H^{1 / 2}(\Omega)} .
$$

\section{REFERENCES}

[1] R. Adams, J. Fournier. Sobolev Spaces. Pure and Applied Mathematics (140), Academic Press, Second Edition, 2003.

[2] M. Anderson. Convergence and Rigidity of Manifolds Under Ricci Curvature Bounds. Invent. Math. 102 (1990), 429-445.

[3] M. Anderson. Degenerations of metric with bounded curvature and applications to critical metrics of Riemannian functionals. Differential Geometry: Riemannian geometry (1990), Proc. Symp. Pure Math. 54 (1993), 53-79.

[4] M. Anderson. Cheeger-Gromov theory and applications to general relativity. The Einstein equations and the large scale behavior of gravitational fields, 347-377, Birkhäuser, Basel, 2004.

[5] M. Anderson, A. Katsuda, Y. Kurylev, M. Lassas, M. Taylor. Boundary Regularity for the Ricci equation, Geometric Convergence, and Gel'fand's Inverse Boundary Problem. Invent. Math. 158 (2004), 261-321.

[6] J. Cheeger. Finiteness Theorems for Riemannian Manifolds. Am. J. Math. 92 (1970), 61-74.

[7] S. Czimek. The localised bounded $L^{2}$-curvature theorem. submitted.

[8] L. Gao. $L^{n / 2}$-Curvature Pinching. J. Diff. Geometry 32 (1990), 713-774. 
[9] D. Gilbarg, N. Trudinger. Elliptic partial differential equations of second order. Reprint of the 1998 edition. Classics in Mathematics. Springer, 2001.

[10] P. Grisvard. Elliptic problems in non-smooth domains. Pitman Advanced Publishing Program, 1985.

[11] R. Greene, H. Wu. Lipschitz Convergence of Riemannian Manifolds. Pac. J. Math. 131 (1988), 119141.

[12] M. Gromov, J. Lafontaine, P. Pansu. Structures métriques pour les variétés riemanniennes. CedicFernand Nathan, Paris, 1981.

[13] S. Kodani. Convergence theorem for riemannian manifolds with boundary. Compositio Mathematica 75 (1990), 171-192.

[14] S. Klainerman, I. Rodnianski. Causal geometry of Einstein-Vacuum spacetimes with finite curvature flux. Journal

[15] D. Maxwell. Rough solutions of the Einstein constraint equations. J. Reine Angew. Math. 590 (2006), 1-29.

[16] R. Perales. Volumes and limits of manifolds with Ricci curvature and mean curvature bounds. Differential Geometry and its Applications, 48 (2016), 23-37.

[17] R. Perales. A survey on the convergence of manifolds with boundary. Contemp. Math. 657 (2016), 179-188.

[18] R. Perales, C. Sormani. Sequences of open Riemannian manifolds with boundary. Pacific J. Math. 270 (2014), no. 2, 423-471.

[19] S. Peters. Convergence of Riemannian Manifolds. Compositio Mathematica 62 (1987), 3-16.

[20] P. Petersen. Convergence Theorems in Riemannian Geometry. Comparison Geometry, 30 (1997), MSRI Publications, 167-202.

[21] P. Petersen. Riemannian Geometry. Third edition, Graduate Texts in Mathematics, 171, Springer, 2016.

[22] M. Reed, B. Simon. Methods of Modern Mathematical Physics: Fourier Analysis, Self-Adjointness. First edition, Academic Press, 1975.

[23] C. Sormani. How Riemannian Manifolds Converge: A Survey. Progress in Mathematics 267: Metric and Differential Geometry, Birkhäuser, 2012.

[24] E. Stein. Singular Integrals and Differentiability Properties of Functions. Princeton University Press, 1970.

[25] J. Szeftel. Parametrix for wave equations on a rough background III: space-time regularity of the phase. arXiv:1204.1770, 2012, 276 pages.

[26] M. Taylor. Partial Differential Equations III: Nonlinear equations. Applied Mathematical Sciences, Vol. 117, Second Edition, Springer, 2011.

[27] R. Wald. General relativity. University of Chicago Press, Chicago, IL, 1984.

[28] D. Yang. Convergence of Riemannian manifolds with integral bounds on curvature I. Ann. Sci. École Norm. Sup. 25 (1992), 77-105.

Department of Computer and Mathematical Sciences, University of Toronto at ScarBorough, TORonto, CAnada 